\title{
Auto- and Cross-repression of Three Arabidopsis WRKY Transcription Factors WRKY18, WRKY40, and WRKY60 Negatively Involved in ABA Signaling
}

\author{
Lu Yan - Zhi-Qiang Liu • Yan-Hong Xu • \\ Kai Lu $\cdot$ Xiao-Fang Wang $\cdot$ Da-Peng Zhang
}

Received: 27 April 2012/ Accepted: 8 October 2012/Published online: 15 December 2012

(C) The Author(s) 2012. This article is published with open access at Springerlink.com

\begin{abstract}
Some members of the WRKY transcription factor family are known to be involved in ABA signaling. However, it remains unclear how the WRKY transcription factors cooperate to regulate ABA signaling. In the present study, we showed that three Arabidopsis (A. thaliana) WRKY proteins previously identified as ABA signaling regulators, WRKY18, WRKY40, and WRKY60, directly target the W-box regions in various domains of the promoters of all their own encoding genes WRKY18, WRKY40, and WRKY60, which was evidenced by chromatin immunoprecipitation and gel shift assays. Furthermore, we showed that the three WRKY proteins inhibit expression of all three $W R K Y$ genes, which was evidenced in both an in vivo assay of coexpression of the WRKY proteins with the three $W R K Y$ promoters and expression analysis of the three $W R K Y$ genes in various wrky mutants. Additionally and importantly, we provide new evidence, with three different testing systems, that WRKY18, WRKY40, and WRKY60 are negative, not positive, ABA signaling regulators, and that $\mathrm{ABA}$ treatment represses all three WRKY genes through a mechanism partly independent of the WRKY proteins, in which the response of the WRKY60
\end{abstract}

Electronic supplementary material The online version of this article (doi:10.1007/s00344-012-9310-8) contains supplementary material, which is available to authorized users.

L. Yan · Z.-Q. Liu · Y.-H. Xu

College of Biological Sciences, China Agricultural University, Beijing 100094, China

K. Lu $\cdot$ X.-F. Wang $(\bowtie) \cdot$ D.-P. Zhang $(\bowtie)$

MOE Systems Biology and Bioinformatics Laboratory, School of Life Sciences, Tsinghua University, Beijing 100084, China

e-mail: wangxf@biomed.tsinghua.edu.cn

D.-P. Zhang

e-mail: zhangdp@tsinghua.edu.cn gene to ABA partly requires WRKY18 and WRKY40. These findings describe a mechanism of auto- and crossrepression of the WRKY transcription repressors that suggests a sophisticated mechanism to balance the negative functions of the WRKY transcription repressors in ABA signaling and helps to understand the WRKY-mediated complex events in ABA signaling pathways.

Keywords Arabidopsis thaliana - Abscisic acid signaling · WRKY transcription factor - Transcription repressor - Seed germination $\cdot$ Early seedling growth

\section{Introduction}

The WRKY-domain transcription factors belong to a superfamily of proteins with the conserved WRKY amino acid sequence at the $\mathrm{N}$-terminus, which interacts with its cognate DNA binding site, the W box (TTGACY), in the promoters of their target genes (Rushton and others 1996; Eulgem and others 2000; Ciolkowski and others 2008). The WRKY transcription factor family in Arabidopsis (Arabidopsis thaliana) consists of more than 74 members, which are divided into three subfamilies according to the number of WRKY domains and category of zinc-finger, forming integral parts of signaling webs that mediate many cellular processes by means of modification of the expression patterns of their target genes (Ulker and Somssich 2004; Eulgem and Somssich 2007; Rushton and others 2010; Chen and others 2012; Rushton and others 2012). The WRKYdomain transcription factors play an important role in plant response to both biotic and abiotic stress (Jones and Dang 2006; Eulgem and Somssich 2007; Rushton and others 2010). Several WRKY transcription factors have been reported to be implicated in plant drought, cold, and heat 
stress tolerance (Chen and others 2012; Rushton and others 2012).

Abscisic acid (ABA) is an essential phytohormone that regulates plant development and plant adaptation to environmental stresses (reviewed in Finkelstein and Rock 2002; Adie and others 2007; Cutler and others 2010). It has been suggested that the WRKY transcription factors play important roles in ABA signal transduction (Jiang and $\mathrm{Yu}$ 2009; Ren and others 2010; Shang and others 2010; Chen and others 2010, 2012; Rushton and others 2012), and some of the Arabidopsis WRKY transcription factors have been identified as key components in the ABA signaling pathways (Jiang and Yu 2009; Ren and others 2010; Shang and others 2010; Chen and others 2010, 2012; Rushton and others 2012). Three homologous WRKY transcription factors, WRKY18, WRKY40, and WRKY60, were shown to cooperate to regulate plant defense in Arabidopsis in a complex pattern of overlapping, antagonistic, and distinct roles in response to different types of microbial pathogens (Xu and others 2006). In a previous report, we showed that these three WRKY proteins are also involved in ABA signaling as negative regulators, where WRKY40 plays a central role. The WRKY40 transcription factor functions as a central transcription repressor directly downstream of a candidate ABA receptor $\mathrm{CHLH} / \mathrm{ABAR}$ (Mg-chelatase $\mathrm{H}$ subunit/putative ABA receptor) that is localized to the chloroplast/plastid envelope membrane (Shen and others 2006; Shang and others 2010). At the same time, an independent group reported that WRKY40 is a transcription repressor and negative regulator in ABA signaling (Chen and others 2010), which is consistent with our findings. However, in contrast to our findings (Shang and others 2010), the group found that two other homologs, WRKY18 and WRKY60, may be transcription activators that are positively involved in ABA signaling (Chen and others 2010). Thus, it is controversial whether WRKY18 and WRKY60 negatively regulate ABA signaling, and also it remains unclear how the three homologous WRKY transcription factors cooperate to regulate ABA signaling.

It is known that the WRKY proteins control the complex mechanisms of transcriptional reprogramming via proteinprotein interaction and autoregulation or cross-regulation (Xu and others 2006; Rushton and others 2010, 2012; Chen and others 2012). In this way, they may function as negative or positive regulators of cell signaling in different physiological processes (Eulgem and others 2000; Eulgem and Somssich 2007; Pandey and Somssich 2009; Chen and others 2012; Rushton and others 2012). In the present study, we showed that three Arabidopsis WRKY proteins, WRKY18, WRKY40, and WRKY60, directly target, and repress, their own encoding $W R K Y$ genes. Additionally and importantly, we provided new evidence that WRKY18, WRKY40, and WRKY60 are negatively, not positively, involved in ABA signaling. We found that $\mathrm{ABA}$ represses all three $W R K Y$ genes through a mechanism partly independent of the WRKY proteins. These findings describe a mechanism of auto- and cross-repression of the three WRKY transcription repressors and help to understand WRKY-mediated complex events in ABA signaling pathways.

\section{Materials and Methods}

\section{Plant Materials and Growth Conditions}

Arabidopsis thaliana ecotype Columbia (Col-0) was used in the generation of transgenic plants. The wrky40-1 (stock No. ET5883, with Ler ecotype as background) was obtained from Cold Spring Harbor Laboratory gene and enhancer trap lines; it contains a Ds transposon inserted within the second exon of WRKY40 (Arabidopsis genomic locus tag: At1g80840). The wrky40-1 mutation was transferred from its background Ler ecotype into Col ecotype by backcrossing as previously described (Shang and others 2010). The wrkyl8-1 (SALK_093916) and wrky60-1 (SALK_120706) are T-DNA insertion knockout mutants with a T-DNA insertion within the first exon in WRKY18 (At4g31800) and WRKY60 (At2g25000) genes, respectively. Both mutants were isolated from the Col ecotype. All three mutants were previously identified as null alleles in their respective genes (Xu and others 2006; Shang and others 2010). All the double and triple mutants were generated by genetic crosses and identified by PCR genotyping.

Plants were grown in a growth chamber at $19-20{ }^{\circ} \mathrm{C}$ on Murashige-Skoog (MS) medium (Sigma) at about $80 \mu \mathrm{mol}$ photons $\mathrm{m}^{-2} \mathrm{~s}^{-1}$ or in compost soil at about $120 \mu \mathrm{mol}$ photons $\mathrm{m}^{-2} \mathrm{~s}^{-1}$ over a $16 \mathrm{~h}$ photoperiod.

\section{Protein Production in E. coli}

We produced the proteins of the full-length WRKY18, WRKY40, and WRKY60 in E. coli essentially as described previously (Shang and others 2010). Briefly, the cDNAs encoding these proteins were amplified by PCR. To isolate the full-length open reading frame of WRKY18, WRKY40, and WRKY60, an EcoRI restriction site was introduced into the forward primers and a $S a l I$ restriction site was introduced into the reverse primers, which are as follows: forward primer $5^{\prime}-\mathrm{G}$ GAATTCTATGGACGGTTCTTCGTTTCTC- $3^{\prime}$ and reverse primer 5'-ACGCGTCGACTCATGTTCTAGATTGCTCC-3' for WRKY18; forward primer 5'-CCGGAATTCTATGGAT CAGTACTC-3' and reverse primer 5'-ACGCGTCGACC TATTTCTCGGTAT- $3^{\prime}$ for WRKY40; and forward primer 5'-GGAATTCTATGGACTATGATCCCAACACC-3' and 
reverse primer $5^{\prime}$-ACGCGTCGACTCATGTTCTTGAAT GCTCTATC- $3^{\prime}$ for WRKY60. The PCR products were digested and cloned into the $\mathrm{pET} 48 \mathrm{~b}(+)$ vector. The fragments in the plasmids were sequenced to check for errors. The recombinant cDNAs were expressed in E. coli BL21 (DE3) (Novagen) strains as $6 \times$ His-tagged fusion proteins. The $E$. coli strains containing the expression plasmids were grown at $37{ }^{\circ} \mathrm{C}$ in $1 \mathrm{~L}$ of the LB medium containing $50 \mu \mathrm{g} /$ $\mathrm{mL}$ kanamycin until the $\mathrm{OD}_{600}$ of the cultures was $0.6-0.8$. Protein expression was induced by the addition of isopropyl $\beta$-D-thiogalactopyranoside to a final concentration of $1 \mathrm{mM}$ at $16{ }^{\circ} \mathrm{C}$ with 150 rotations per minute. After $16 \mathrm{~h}$, the cells were lysed and proteins purified on a $\mathrm{Ni}^{2+}$-chelating column as described in the pET system manual.

Effects of ABA Treatment on Expression of WRKY18, WRKY40, and WRKY60

Three-day-old young seedlings were transferred to the medium supplemented with ABA at indicated concentrations and continued to grow for 2 weeks before sampling for assaying gene expression by real-time PCR. Threeweek-old mature plants were also used to investigate the effects of ABA. Plants were sprayed with ABA solutions at indicated concentrations and sampled at different time intervals for analysis.

\section{Quantitative Real-Time PCR}

Real-time PCR for gene expression was performed as previously described (Wu and others 2009), essentially according to the instructions provided for the BioRad RealTime System CFX96TM C1000 Thermal Cycler (Singapore). The gene-specific primers used were as follows: forward primer 5'-AGACAACCCGTCACCT-3' and reverse primer $5^{\prime}$-GCATCGTATTATCCCTTT-3' for $W R K$ $Y 18$, forward primer $5^{\prime}$-CTCCCAAGAAACGCAA-3' and reverse primer 5'-GCAACTAACACGGACTGA-3' for WRKY40, and forward primer $5^{\prime}$-TTTTCACCGTCTTGTCT- $3^{\prime}$ and reverse primer 5'-ATGCTCTATCAATCTCCC- $3^{\prime}$ for WRKY60. Total RNA was isolated with the RNasy plant mini kit (Qiagen) supplemented with an oncolumn DNA digestion (Qiagen RNase-Free DNase set) according to the manufacturer's instructions, and then the RNA sample was reverse transcribed with the Superscript II RT kit (Invitrogen) in $25 \mathrm{~mL}$ volume at $42{ }^{\circ} \mathrm{C}$ for $1 \mathrm{~h}$. Amplification of ACTIN2/8 genes was used as an internal control (with forward primer $5^{\prime}$-GGTAACATTGTGCTCA GTGGTGG-3' and reverse primer 5'-AACGACCTTA ATCTTCATGCTGC- $3^{\prime}$ ). The suitability of the oligonucleotide sequences in terms of efficiency of annealing was evaluated in advance using the Primer 5.0 program. The cDNA was amplified using SYBR Premix Ex Taq (TaKaRa) with a DNA Engine Opticon 2 thermal cycler in $10 \mathrm{~mL}$ volume and the following program: one cycle of $95{ }^{\circ} \mathrm{C}$ for $10 \mathrm{~s}$ and 40 cycles of $94{ }^{\circ} \mathrm{C}$ for $5 \mathrm{~s}$ and $60^{\circ} \mathrm{C}$ for $30 \mathrm{~s}$. The amplification of the target genes was monitored every cycle by SYBR Green fluorescence. The Ct (threshold cycle), defined as the PCR cycle at which a statistically significant increase of reporter fluorescence was first detected, was used as a measure for the starting copy numbers of the target gene. Relative quantitation of the target gene expression level was obtained using the comparative $\mathrm{Ct}$ method. Three technical replicates were performed for each experiment. For all the quantitative real-time PCR analysis, the assays were repeated three times along with at least three independent repetitions of the biological experiments, and the means of the three biological experiments were calculated for estimating gene expression.

Analysis of Gene Expression by Promoter-GUS

Transformation

The promoter fragments of Arabidopsis gene At1g 80840 (WRKY40), At4g31800 (WRKY18), and At2g25000 (WRKY60) were amplified by PCR. The primers used were as follows: forward primer $5^{\prime}$-AACTGCAGTGCCGACC TACAAATC- $3^{\prime}$ and reverse primer $5^{\prime}$-CGGGATCCATC TTAAGATACAAACCAA- $3^{\prime}$ for a 1,260-bp promoter fragment (upstream of ATG) of WRKY18, forward primer 5'-AACTGCAGAGCCGTGTGGGCTTGACTTT-3' and reverse primer 5'-CGGGATCCCGGTGGATCTTCTTCA ACTCG-3' for a 1,098-bp promoter fragment (upstream of ATG) of WRKY40, and forward primer 5'-ACTGCA GTTTGCTGCTGTTTCAAG-3' and reverse primer $5^{\prime}-\mathrm{CG}$ GGATCCAAATTTAGGTTCACAGGAG- $3^{\prime}$ for a 1,346 bp promoter fragment (upstream of ATG) of WRKY60. The DNA fragments were cloned into the pCAMBIA1300-221 vector and introduced into the GV3101 strain of Agrobacterium tumefaciens and transformed into Arabidopsis wildtype (Col-0) plants or wrky40 mutant or wrky18 mutant or wrky60 mutant plants by floral infiltration. T3 generation homologous plants were used for the analysis of GUS ( $\beta$-glucuronidase) activity. GUS staining was performed essentially according to Jefferson and others (1987). Whole plants or tissues were immersed in $1 \mathrm{mM}$ 5-bromo-4chloro-3-indolyl-b-GlcUA (X-gluc) solution in $100 \mathrm{mM}$ sodium phosphate ( $\mathrm{pH} 7.0$ ), $2 \mathrm{mM}$ EDTA, $0.05 \mathrm{mM}$ ferricyanide, $0.05 \mathrm{mM}$ ferrocyanide, and $0.1 \%$ (v/v) Triton $\mathrm{X}-100$ for $5-6 \mathrm{~h}$ at $37^{\circ} \mathrm{C}$. Chlorophyll was cleared from the tissues with a mixture of $30 \%$ acetic acid and $70 \%$ ethanol. 
Chromatin Immunoprecipitation (ChIP) Assay

ChIP assay was performed as previously described (Saleh and others 2008; Shang and others 2010). Two-week-old seedlings were sampled for the assays. The WRKY40-specific antibody against WRKY40 ${ }^{\mathrm{N}}$ (an N-terminal-truncated form of WRKY40), produced as previously described (Shang and others 2010), was used for the ChIP assay. The primers used for PCR amplification for different promoters are listed in Supplementary Table 1. PCR amplification was performed using 35 cycles at $56^{\circ} \mathrm{C}$ for WRKY18-, WRKY40-, and WRKY60-promoter fragments. Aliquots of the PCR reactions were resolved by electrophoresis on a $1 \%$ agarose gel. Images of the ethidium bromide-stained gels were captured by the Molecular Imager System (Gel Doc XR, BioRad) with ImageQuant software (Molecular Dynamics). The results presented here are from at least five independent experiments.

To determine quantitatively the WRKY40-DNA (target promoters) binding, real-time PCR analysis was performed according to the procedure described previously with the Actin2 3 -untranslated region sequence as the endogenous control (Mukhopadhyay and others 2008; Shang and others 2010). The relative quantity value calculated by the $2-\mathrm{ddCt}$ method is reported as DNA binding ratio (differential site occupancy). The same primers used for the above-mentioned PCR analysis were used for the real-time PCR. A fragment of the Actin2 promoter was used as a negative control, and the primers used were (forward primer) $5^{\prime}$-CGTTTCGCTTTCCT-3' and (reverse primer) $5^{\prime}$-AAC GACTAACGAGCAG- $3^{\prime}$.

\section{Gel Shift Assay}

Gel shift assay (GSA) was performed using recombinant His-WRKY40, His-WRKY18, and His-WRKY60 protein purified from E. coli as previously described (Shang and others 2010). The promoter fragments used for the GSA were amplified by PCR using the following primer pairs: forward primer 5'-GAAGAGGATATAAAGGGAAC-3' and reverse primer 5'-CGAAAAATCAATAATTTCGG C-3' for the first fragment of the WRKY18 promoter (pWRKY18-1; -1171 to $-1065,107 \mathrm{bp}$ ); forward primer $5^{\prime}$-CAGTGACTAAAGCAGAAT- $3^{\prime}$ and reverse primer $5^{\prime}$-TACGATGAAGGTGACAAA-3' for the second fragment of the WRKY18 promoter (pWRKY18-2; -904 to -734, $171 \mathrm{bp}$ ); forward primer $5^{\prime}$-AATCTTAAATTTGACCTT AG- $3^{\prime}$ and reverse primer 5'-TTCGGTCACAAGTTGGT C- $3^{\prime}$ for the third fragment of the WRKY18 promoter (pWRKY18-3; -258 to $-67,192 \mathrm{bp}$ ); forward primer $5^{\prime}-\mathrm{A}$ GCCGTGTGGGCTTGACTTTG- $3^{\prime}$ and reverse primer 5'-GCACGTTTGACAAAAAAAATGAC-3' for the first

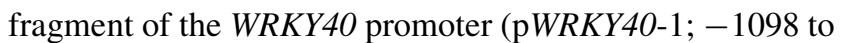

-939, $160 \mathrm{bp}$ ); forward primer 5'-CCATTTCTTTTATC CCAC- $3^{\prime}$ and reverse primer $5^{\prime}$-CATCTTAGATTTTTCA GAC $-3^{\prime}$ for the second fragment of the WRKY4O promoter (pWRKY40-2; -855 to $-724,132 \mathrm{bp}$ ); forward primer 5'-CCCGACAGAAATGTCAAC-3' and reverse primer $5^{\prime}$-TTTTGGATAGTTGGTTG-3' for the third fragment of the WRKY40 promoter (pWRKY40-3; -545 to $-415 ; 131$ $\mathrm{bp}$ ); forward primer $5^{\prime}$-TCCCTAACCAACTTGACA- $3^{\prime}$ and reverse primer $5^{\prime}$-ACTATGGAAACAGAGGCT- $3^{\prime}$ for the first fragment of the WRKY60 promoter (pWRKY60-1; -1296 to $-1098,199 \mathrm{bp}$ ); forward primer 5'-TAAAAA AGATGACAAAACAA- $3^{\prime}$ and reverse primer $5^{\prime}$-AGTTGC ATTAGTTAATTATGTCA- $3^{\prime}$ for the second fragment of the WRKY60 promoter (pWRKY60-2; -965 to $-854,112$ bp); forward primer 5'-GGGCATAGTCAATGGGTC-3' and reverse primer $5^{\prime}$-CGGAGAAAACAAGTGTAAAG-3' for the third fragment of the WRKY60 promoter ( $\mathrm{pWRKY60-}$ 3 ; -793 to $-652,142 \mathrm{bp}$ ); and forward primer $5^{\prime}$-CAACT TGCTTTTTGTCAAT- $3^{\prime}$ and reverse primer $5^{\prime}$-TGTAA TTCTAATTGTGCC-3' for the fourth fragment of the WRKY60 promoter ( $\mathrm{p} W R K Y 60-4 ;-534$ to $-375,160 \mathrm{bp}$ ).

The site-specific mutations from GTCA to CTCA or from TGAC to TTAC in the core sequence of the W-box of the WRKY40, WRKY18, and WRKY60 promoters were introduced into the above promoters by two independent PCRs with the following primers (with the mutated W-box underlined), in addition to the above-mentioned primers for each promoter: forward primer 5'-GAAATTTATGTATTACGTCCAAATT TTTC- $3^{\prime}$ and reverse primer 5'-GAAAAATTTGGACGTAA TACATAAATTTC- $3^{\prime}$ for mutated pWRKY18-1; forward primer 5'-GTACCACCTAACAGTTACTAAAGCAGAAT-3' and reverse primer 5'-TACGATGAAGGTGAGAAATA

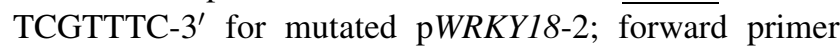
5'-AATCTTAAATTTTACCTTAGGATAA- ${ }^{\prime}$ and reverse primer 5'-TTCGGTCACAAGTTGGTC-3' for mutated pWRKY18-3; forward primer $5^{\prime}$-AGCCGTGTGGGCTTTA CTTTTACCGAACAAGGCA-3' and reverse primer $5^{\prime}-\mathrm{GC}$ ACGTTTGAGAAAAAAAATGAGAACTAGTTGGTG-3' for mutated $\mathrm{pW} R K Y 40-1$; forward primer 5'-CCATTTCTT TTATCCCACATCTCATTTGTAGTCC- $3^{\prime}$ and reverse primer 5'-CTTGATGCATGGAGTGAGAAAAAAAAAG ATAAC- $3^{\prime}$ for mutated $\mathrm{pWR} K Y 40-2$; forward primer $5^{\prime}-\mathrm{CC}$ CGACAGAAATCTCAACCAACTATCCA- $3^{\prime}$ and reverse primer 5'-TTTTGGATAGTTGGTTGAGATTCTT-3' for mutated pWRKY40-3; forward primer 5'-TCCCTAACCAACTTTACAATTCGAATAATG-3' and reverse primer 5'-ACTATGGAAACAGAGGCT- $3^{\prime}$ for mutated $\mathrm{pWRKY}$ 60-1; forward primer 5'-TAAAAAAGATTACAAAACAA AAAAT- $3^{\prime}$ and reverse primer $5^{\prime}$-AGTTGCATTAGTTAA TTATGTAAGCTAGGCA-3' for mutated pWRKY60-2; forward primer 5'-GGGCATACTCAATGGCTCAACTCA AGCGAAAG- $3^{\prime}$ and reverse primer 5'-CGGAGAAAACAAGTGTAAAG- $3^{\prime}$ for mutated $\mathrm{pWR}$ KY60-3; and for 
ward primer 5'-CAACTTGCTTTTTCTCAATACGTATT AAAT- $3^{\prime}$ and reverse primer $5^{\prime}$-TGTAATTCTAATTGTG

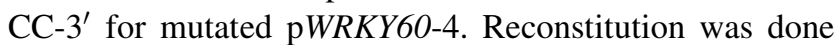
using equimolar quantities of the two fragments from the initial PCRs for each promoter, which were used as templates of a third PCR. The mutations were verified by sequence analysis. Each of the promoter fragments was labeled in the base $\mathrm{T}$ with digoxigenin-dUTP (Roche) according to the manufacturer's instructions. Binding reactions were performed as previously described (Shang and others 2010) using $50 \mathrm{ng}$ of His-WRKY40, HisWRKY18, or His-WRKY60 fusion protein and $26 \mathrm{ng}$ for each of digoxigenin-labeled promoter fragments. Competition experiments were performed using from 5-molar excess of unlabeled fragments.

Trans-inhibition of WRKY18, WRKY40, and WRKY60

Promoter Activity by WRKY40, WRKY18,

or WRKY60 in Tobacco Leaves

This assay was performed essentially as previously described (Shang and others 2010). WRKY18, WRKY40, and WRKY60 were used for the effector constructs. The cDNAs were amplified by PCR using the forward primer 5'-CGCGGATCCATGGACGGTTCTTCGTTTCTC- ${ }^{\prime}$ ' and reverse primer $5^{\prime}$-CCGCTCGAGTCATGTTCTAGATTG CTCC- $3^{\prime}$ for WRKY18, the forward primer $5^{\prime}$-CGC GGATCCATGGATCAGTACTCAT- $3^{\prime}$ and reverse primer 5'-CCGCTCGAGCTATTTCTCGGTATGA-3' for WRK $Y 40$, and the forward primer $5^{\prime}$-CGCGGATCCATGGACTATGATCCCAACACC- $3^{\prime}$ and reverse primer $5^{\prime}$-CCGCT CGAGTCATGTTCTTGAATGCTCTATC- $3^{\prime}$ for WRKY 60. The PCR products were fused to the pBI121 vector downstream of the CaMV $35 \mathrm{~S}$ promoter at the BamHI/XhoI sites. Reporter constructs were composed of the WRKY18, WRKY40, and WRKY60 promoters linked to LUC (cDNA encoding luciferase). The WRKY promoters were isolated using following primers: forward primer $5^{\prime}$-GG GGTACCTGCCGACCTACAAATC- $3^{\prime}$ and reverse primer $5^{\prime}$-TCCCCCGGGATCTTAAGATACAAACCAA-3' $(1,260$ bp) for WRKY18 promoter, forward primer 5'-GGGGTACCAGCCGTGTGGGCTTGACTTT-3' and reverse primer $5^{\prime}$-TCCCCCGGGGTAAATATATGTA GGATGAATC-3' (1,098 bp) for WRKY40 promoter, and forward primer 5'-GGGGTACCTTTGCTGCTGTTT CAAG- $3^{\prime}$ and reverse primer $5^{\prime}$-TCCCCCGGGAAATT TAGGTTCACAGGAG-3' (1,346 bp) for WRKY60 promoter. The $L U C$ cDNA was PCR-amplified using the forward primer $5^{\prime}$-TCCCCCGGGATGGAAGACGCCAAA AAC- $3^{\prime}$ and reverse primer $5^{\prime}$-CGGGATCCTTACACGG CGATCTTTCCGC-3' from pGL3-Basic Vector harboring the LUC cDNA. The DNA sequences of WRKY40, WRKY18, and WRKY60 promoters were separately fused to the
KpnI/SmaI sites of the pCAMBIA1300 vector from which the CaMV 35S promoter was deleted, with the $L U C$ cDNA fused to the SmaI/BamHI sites downstream of the WRKY40, WRKY18, and WRKY60 promoters. The constructs were mobilized into A. tumefaciens strain GV3101. Bacterial suspensions were infiltrated into young but fully expanded leaves of the 7-week-old $N$. benthamiana plants using a needleless syringe. It is noteworthy that the amounts of the constructs were the same among treatments and controls for each group of assay. After infiltration, plants were grown first under dark conditions for $12 \mathrm{~h}$ and then with $16 \mathrm{~h}$ light/day for $60 \mathrm{~h}$ at room temperature, and the LUC activity was observed with a CCD imaging apparatus (iXon, Andor). The experiments were repeated independently at least five times with similar results.

\section{Protein Extraction and Immunoblotting}

The leaves of tobacco or Arabidopsis were sampled and frozen in liquid nitrogen, ground in a prechilled mortar with a pestle to a fine powder, and transferred to a $1.5 \mathrm{~mL}$ tube. The extraction buffer consisted of $50 \mathrm{mM}$ Tris- $\mathrm{HCl}$ (pH 7.8), $50 \mathrm{mM} \mathrm{NaCl}, 0.1 \%$ (v/v) Tween-20, $10 \%(\mathrm{v} / \mathrm{v})$ glycerol, $0.15 \%(\mathrm{v} / \mathrm{v}) 2$-mercaptoethanol, and $5 \mu \mathrm{g} / \mathrm{mL}$ protein inhibitor cocktail (Roche). The extraction buffer was added to the tube (buffer:sample $=2: 1$ ). The sample was extracted for $3 \mathrm{~h}$ in ice. The extracts were centrifuged for $20 \mathrm{~min}$ at $16,000 \mathrm{~g}$. Then the supernatant was transferred to a new Eppendorf tube and centrifuged again at $16,000 \mathrm{~g}$ for $20 \mathrm{~min}$, and then concentrate the supernatant. The SDS-PAGE and immunoblotting assays were performed according to our previously described procedures (Wu and others 2009; Shang and others 2010). The specific antibodies against LUC were purchased from SANTA (product No. sc-74548). The WRKY40-specific antibody against WRKY $40^{\mathrm{N}}$ (an N-terminal-truncated form of WRKY40), produced as previously described (Shang and others 2010), was used for detecting WRKY40 protein.

\section{Phenotypic Analysis}

Phenotypic analysis was done as previously described (Shen and others 2006; Wu and others 2009; Shang and others 2010). For germination, seeds were planted on MS medium (Sigma product No. M5524; full-strength MS) containing $3 \%$ sucrose and $0.8 \%$ agar (pH 5.9). The medium was supplemented with different concentrations of $( \pm) \mathrm{ABA}$ as indicated. The seeds were incubated at $4{ }^{\circ} \mathrm{C}$ for 3 days for stratification and then placed under light conditions at $20{ }^{\circ} \mathrm{C}$; germination (emergence of radicals) was scored at the indicated times. Early seedling growth was assessed using two techniques. One way was to plant the seeds directly in the $( \pm)$ ABA-containing MS medium. 
They were then incubated at $4{ }^{\circ} \mathrm{C}$ for 3 days for stratification and then placed under light conditions at $20^{\circ} \mathrm{C}$ to investigate the response of seedling growth to $\mathrm{ABA}$ after germination. The other technique was to transfer germinating seeds to $( \pm) \mathrm{ABA}$-containing MS medium as follows: Seeds were germinated after stratification on common MS medium and then transferred to MS medium supplemented with different concentrations of $( \pm) \mathrm{ABA}$ in the vertical position. The time for transfer was $48 \mathrm{~h}$ or 4 days (as indicated) after stratification. Seedling growth was investigated after the transfer at the indicated times, and the length of the primary roots was measured using a ruler.

\section{Accession Numbers}

Sequence data from this article can be found in the Arabidopsis Genome Initiative database under the following accession numbers: At1g80840 (WRKY40), At4g31800 (WRKY18), At2g25000 (WRKY60), and At2g36270 (ABI5). Germplasm identification numbers for mutant lines and SALK lines are as follows: wrky40-1 (stock No. ET5883, Cold Spring Harbor Laboratory gene and enhancer trap lines), wrky18-1 (ABRC stock No. SALK_093916), and wrky60-1 (ABRC stock No. SALK_120706).

\section{Results}

WRKY18, WRKY40, and WRKY60 Target Each of the Three WRKY Genes

It has been reported that WRKY18 and WRKY40 bind the promoter of the WRKY60 gene at a cluster of three W-box sequences between -791 and -773 relative translation start codon (Chen and others 2010), which corresponds to the fourth to sixth W-boxes numbered in the present study (see Fig. 1a). However, it remains unknown whether these two WRKYs bind the promoter of the WRKY60 gene at the other sites, namely, the first to third and the seventh to ninth (Fig. 1a), and whether the three WRKYs bind mutually the promoters of their encoding genes, including their own genes. A search of the Arabidopsis genomic sequence showed that all three WRKY genes have in their promoter region several $\mathrm{W}$-box sequences, the core of a cis-element to which the WRKY transcription factors bind (Fig. 1a), which suggests that the WRKY proteins could bind the promoters of these WRKY genes. With chromatin coimmunoprecipitation (ChIP) analysis combined with PCR and quantitative real-time PCR, we showed that WRKY40 binds the promoters of WRKY18 and WRKY60 and its own encoding gene WRKY4O via the core W-box sequence TGAC at different sites (Fig. 1b; Supplementary Table 1), which covers the second to fourth W-boxes (Fig. 1a, b, pWRKY18-2 and pWRKY18-3 domains) for the WRKY18 promoter, the fifth to tenth W-boxes (Fig. 1a, b, pWRKY40-2 and pWRKY40-3 domains) for the WRKY4O promoter, and the first (Fig. 1a, b, pWRKY60-1 domain) and fourth to seventh W-boxes (Fig. 1a, b, pWRKY60-3 and $\mathrm{pWRKY60-4}$ domains) for the WRKY60 promoter. The pWRKY60-3 domain covers the cluster of three W-box sequences between -791 and -773 (the fourth to sixth W-boxes) in the WRKY60 promoter, so the binding of WRKY40 to the pWRKY60-3 domain is consistent with data in the previous report (Chen and others 2010). The WRKY40 protein does not bind the pWRKY18-1 domain covering the first W-box in the WRKY18 promoter, or the $\mathrm{p} W R K Y 40-1$ domain covering the first to fourth $\mathrm{W}$-boxes in the WRKY4O promoter, or the pWRKY60-2/5 domains covering the second, third, eighth, and ninth W-boxes in the WRKY60 promoter (Fig. 1a-c).

Furthermore, we tested, using a gel shift assay, if all three WRKY proteins interact with the promoters of each of the three WRKY genes (Fig. 2). As shown in Fig. 2b, we first confirmed the binding of the WRKY40 protein to different sites of the promoters of the three $W R K Y$ genes as described above (Fig. 1). We further observed that the WRKY18 protein binds the promoters of all three WRKY genes, including the promoter of its own encoding gene WRKY18 at two domains (p2 and $\mathrm{p} 3$ ) covering the second to fourth W-boxes (Figs. 1a and 2a), the promoter of the WRKY4O gene at one domain (p3) covering the eighth to tenth W-boxes (Figs. 1a and 2a), and the promoter of the WRKY60 gene at two domains ( $\mathrm{p} 2$ and $\mathrm{p} 3$ ) covering the second to sixth W-boxes (Figs. 1a and 2a). The WRKY18 protein does not bind the $\mathrm{p} 1$ domain covering the first W-box in the WRKY18 promoter, or the $\mathrm{p} 1$ and $\mathrm{p} 2$ domains covering the first to seventh W-boxes in the WRKY40 promoter, or the $\mathrm{p} 1$ domain covering the first $\mathrm{W}$-box in the WRKY60 promoter (data not shown).

The WRKY60 protein, like WRKY18 and WRKY40, also binds the promoters of all three WRKY genes, including the promoter of WRKY18 at two domains (p1 and p3) covering the first and fourth W-boxes (Figs. 1a and 2c), the promoter of WRKY4O at two domains (p1 and p3) covering the first to fourth and eighth to tenth W-boxes (Figs. 1a and 2c), and the promoter of its own encoding gene WRKY60 at two domains ( $\mathrm{p} 1$ and $\mathrm{p} 3$ ) covering the first and the fourth to sixth W-boxes (Figs. 1a and 2c). The WRKY60 protein does not bind the $\mathrm{p} 2$ domain covering the second and third W-boxes in the WRKY18 promoter, or the $\mathrm{p} 2$ domain covering the fifth to seventh $\mathrm{W}$-boxes in the WRKY40 promoter, or the $\mathrm{p} 2$ domain covering the second and third $\mathrm{W}$-boxes in the WRKY60 promoter (data not shown). 

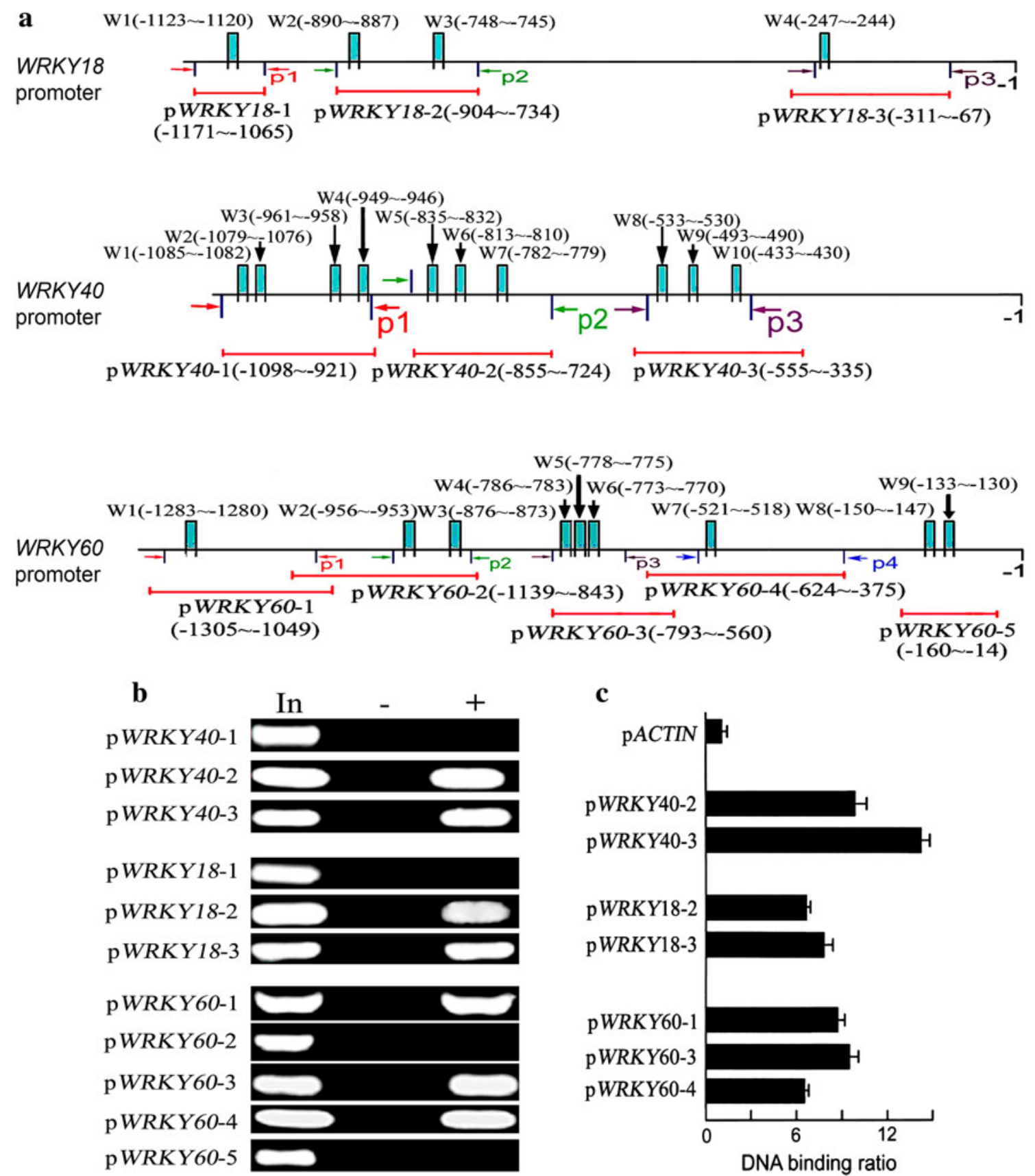

Fig. 1 WRKY40 interacts with the promoters of WRKY18, WRKY40, and WRKY60 genes: ChIP assays. a The promoter structures of WRKY18 (top), WRKY40 (middle), and WRKY60 (bottom) genes. W1, $\mathrm{W} 2$, and so on denote each W-box numbered from left to right with sequence sites relative to the star code (numbers in parentheses). Arrows indicate the sequence fragments (denoted by p1, p2, ...) used in the gel shift assays (GSA) described in Fig. 2. The red bars indicate the different domains or sequence fragments (denoted by

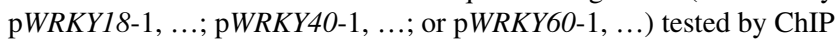
assays described in $\mathbf{b}$ with the numbers in parenthesis indicating locations of the different domains relative to the star code. Note that the numbering of the sequence fragments used in GSA $(\mathrm{p} 1, \mathrm{p} 2, \ldots)$ substantially corresponds to those of the domains used in ChIP assay (pWRKY18-1, ..; pWRKY40-1, ..; or pWRKY60-1, ..). b WRKY40 interacts with the promoters of WRKY18, WRKY40, and WRKY60 genes: PCR data from ChIP assay with the WRKY40-specific antibody against WRKY $40^{\mathrm{N}}$. In the promoter fragment names, the prefix " $p$ " indicates promoter. The sequences for each promoter fragment (indicated by the suffix number) are indicated in panel a and listed in detail in Supplementary Table 1. In, PCR product from the chromatin DNA; -, PCR product from ChIP with preimmune serum (as a negative control); + , PCR product from ChIP with the antibody against WRKY $40^{\mathrm{N}}$. c WRKY40 interacts with the promoters of WRKY18, WRKY40, and WRKY60 genes: real-time PCR data from ChIP assay with the WRKY40-specific antibody against WRKY $40^{\mathrm{N}}$ with the actin promoter (pACTIN) as a negative control. All the symbols for promoters present the same significances as described in panel b 

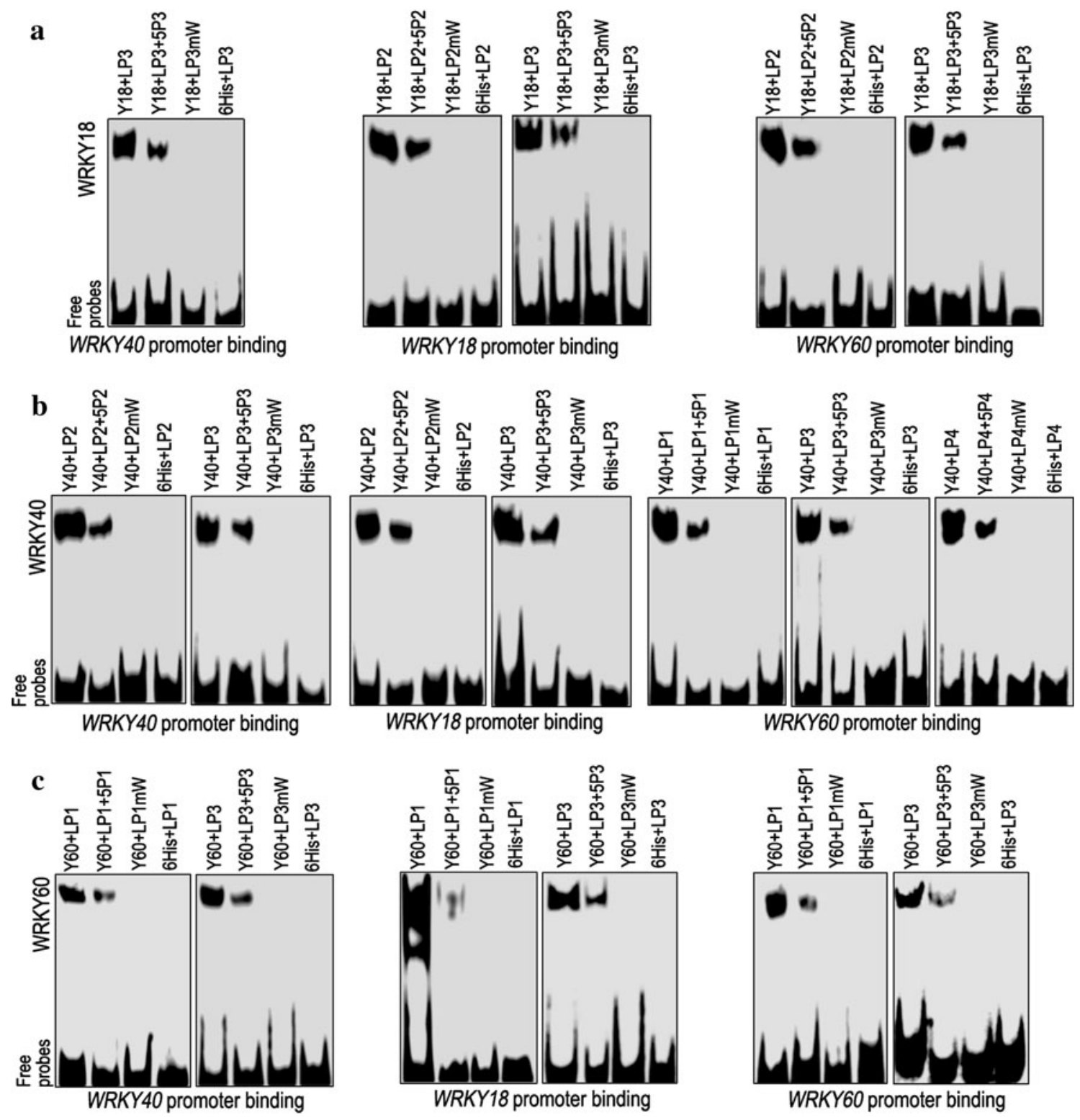

Fig. 2 WRKY18, WRKY40, and WRKY60 interact with each of promoters of their encoding genes: gel shift assays. a WRKY18 binds the promoters of WRKY18 and WRKY60 genes at their $\mathrm{p} 2$ and $\mathrm{p} 3$ domains and the promoter of the WRKY4O gene at its $\mathrm{p} 3$ domain; b WRKY40 binds the promoters of WRKY18 and WRKY40 genes at their $\mathrm{p} 2$ and $\mathrm{p} 3$ domains and the promoter of the WRKY60 gene at its $\mathrm{p} 1, \mathrm{p} 3$, and $\mathrm{p} 4$ domains. c WRKY60 binds the promoters of WRKY18, WRKY40, and WRKY60 genes at their $\mathrm{p} 1$ and $\mathrm{p} 3$ domains. Y18, Y40, and Y60 indicate, respectively, WRKY18, WRKY40, and WRKY60.

As negative controls, no binding activity of the WRKY proteins to $6 \mathrm{His}$ peptide or to the $W R K Y$ promoter domains harboring a mutated W-box was observed, and the binding intensities significantly decreased when adding a fivefold unlabeled probe for competition, showing that the observed binding activity of the WRKY proteins was specific in this GSA system.
LP, labeled probe; LPmW, labeled probe with the mutated W-box as indicated in Supplementary Table 1, which was used as a negative control; 6His, 6His tag peptide fused to the WRKY protein, which was used as another negative control; $\mathrm{P}$, unlabeled probe; 5P, fivefold unlabeled probe addition. The suffix numbers of $\langle\mathrm{P}\rangle$ and $\langle\mathrm{LP}\rangle$ indicate the promoter fragments (the domains $\mathrm{p} 1, \mathrm{p} 2, \ldots$ ) described in Fig. 1a. These promoter fragments were used as the probe, of which the sequences are listed in detail in Supplementary Table 1. The experiments were repeated five times with the same results

WRKY18, WRKY40, and WRKY60 Inhibit Expression of All Three WRKY Genes

We tested if WRKY18, WRKY40, and WRKY60 regulate the promoter activity of the three WRKY genes using an in vivo system by cotransforming tobacco leaves with the $W R K Y$ s and WRKY promoters linked to luciferase (LUC). 
The results showed that all three WRKY proteins interact with each of the three $W R K Y$ promoters in vivo and inhibit significantly the promoter activity reported by LUCproduced fluorescence (Fig. 3a-c). To confirm these observations, we tested the levels of LUC protein, the encoding gene of which was driven by the WRKY promoters. We found that the LUC protein levels significantly decreased when the $W R K Y$-promoter-LUC constructs were coexpressed with 35S-promoter-driven WRKY genes (Fig. 4a-c). The data verify that the WRKY proteins did repress the WRKY promoter activities.

Furthermore, we transformed different wrky mutants harboring corresponding null mutations with each of the three WRKY promoters linked to a reporter GUS and observed that the promoter activities of the three WRKY genes were all significantly enhanced in the wrkyl8, wrky40, and wrky60 mutants (Fig. 5a-c). Also, we assayed expression of the three $W R K Y$ genes in different wrky mutants. The results further showed that the expression level of WRKY18 was enhanced in the wrky40, wrky60 single mutants and in the wrky40 wrky60 double mutant, with a higher extent of upregulation in the wrky60 single mutant similar to that in the wrky40 wrky60 double mutant (Fig. 5a, b). We observed that expression of WRKY4O was enhanced in the wrkyl8, wrky60 single mutants and in the wrky18 wrky60 double mutant, with a higher extent of upregulation in the wrky60 single mutant similar to that in wrky 18 wrky60 double mutant (Fig. 5a, b). The WRKY60 expression was also enhanced by a disruption mutation of the WRKY18 or the WRKY4O gene in the wrkyl8 or the wrky40 mutant, with a higher extent of upregulation in the wrkyl8 wrky40 double mutant (Fig. 5c). These findings reveal that WRKY18, WRKY40, and WRKY60 mutually require each other for repression of their encoding WRKY genes. However, WRKY60 does not function synergistically with WRKY18 to repress the WRKY40 gene, or with WRKY40 to repress the WRKY18 gene, whereas WRK18 and WRKY40 function synergistically to repress WRKY60 expression.

ABA Represses Expression of the Three WRKY Genes in Both Wild-type Plants and wrky Mutants

We assayed the effects of exogenous application of ABA on the expression of the three WRKY genes by treating plants at both early and mature developmental stages. For the early-stage treatment, the germinating seeds were transferred from ABA-free medium to medium containing ABA and sampled for analysis 2 weeks after stratification. This system mimics a system used to assay the post-
Fig. 3 WRKY18, WRKY40, and WRKY60 inhibit the promoter activities of their encoding genes: an in vivo test in tobacco leaves. The tobacco leaves were transformed a with $\mathrm{pWRKY18-LUC}$ alone, pWRKY18-LUC plus WRKY18 (left), pWRKY18-LUC plus WRKY40 (middle), or pWRKY18-LUC plus WRKY60 (right). b with $\mathrm{pWR}$ KY40-LUC alone, $\mathrm{pWRKY40-LUC} \mathrm{plus}$ WRKY18 (left), pWRKY40LUC plus WRKY40 (middle), or $\mathrm{pWRKY40-LUC} \mathrm{plus}$ WRKY60 (right); and c with pWRKY60-LUC alone, pWRKY60-LUC plus WRKY18 (left), $\mathrm{pWRKY60-LUC} \mathrm{plus}$ WRKY40 (middle), or pWRKY60-LUC plus WRKY60 (right). The experiments were repeated five times with the same results a
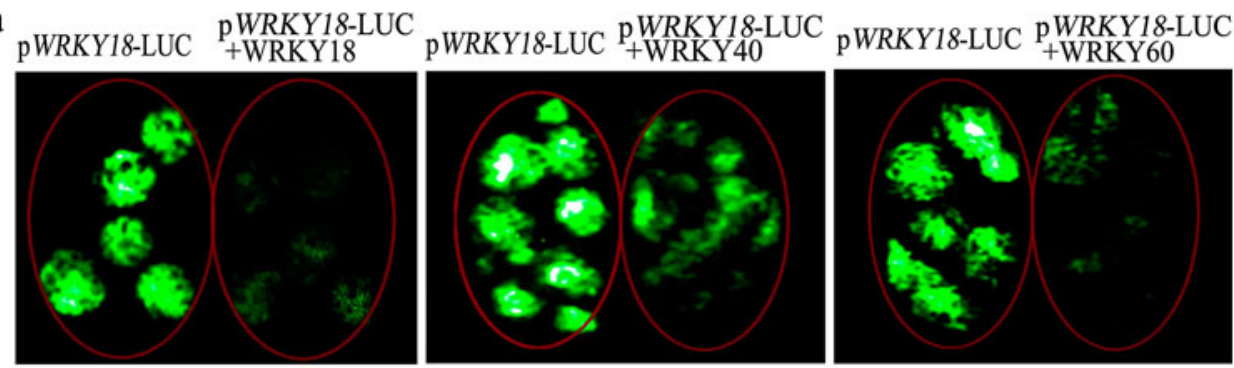

b
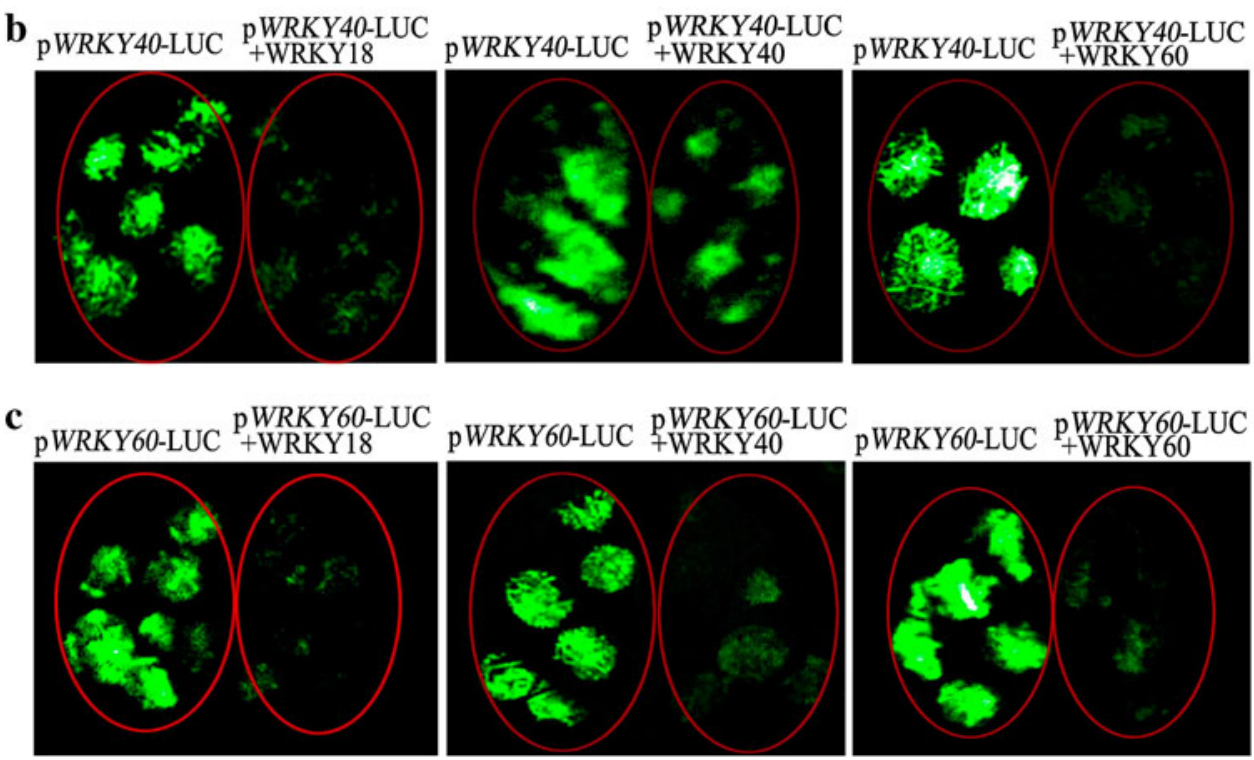
Fig. 4 WRKY18, WRKY40, and WRKY60 inhibit the promoter activities of their encoding genes: confirmation with immunoblotting analysis of LUC in transgenic tobacco leaves. The LUC levels of the transgenic tobacco leaves presented in Fig. 3 were assayed with immunoblotting. Panels a, $\mathbf{b}$, and $\mathbf{c}$ correspond to the same panels in Fig. 3, with the same symbols indicating the treatments. Protein amounts were evaluated by scanning the protein bands, and relative band intensities, normalized relative to the intensity with the value from the sample of the transformation $\mathrm{pWRKY}$-LUC alone (as $100 \%$ ), are indicated by numbers below the bands. The experiments were repeated five times with the similar results

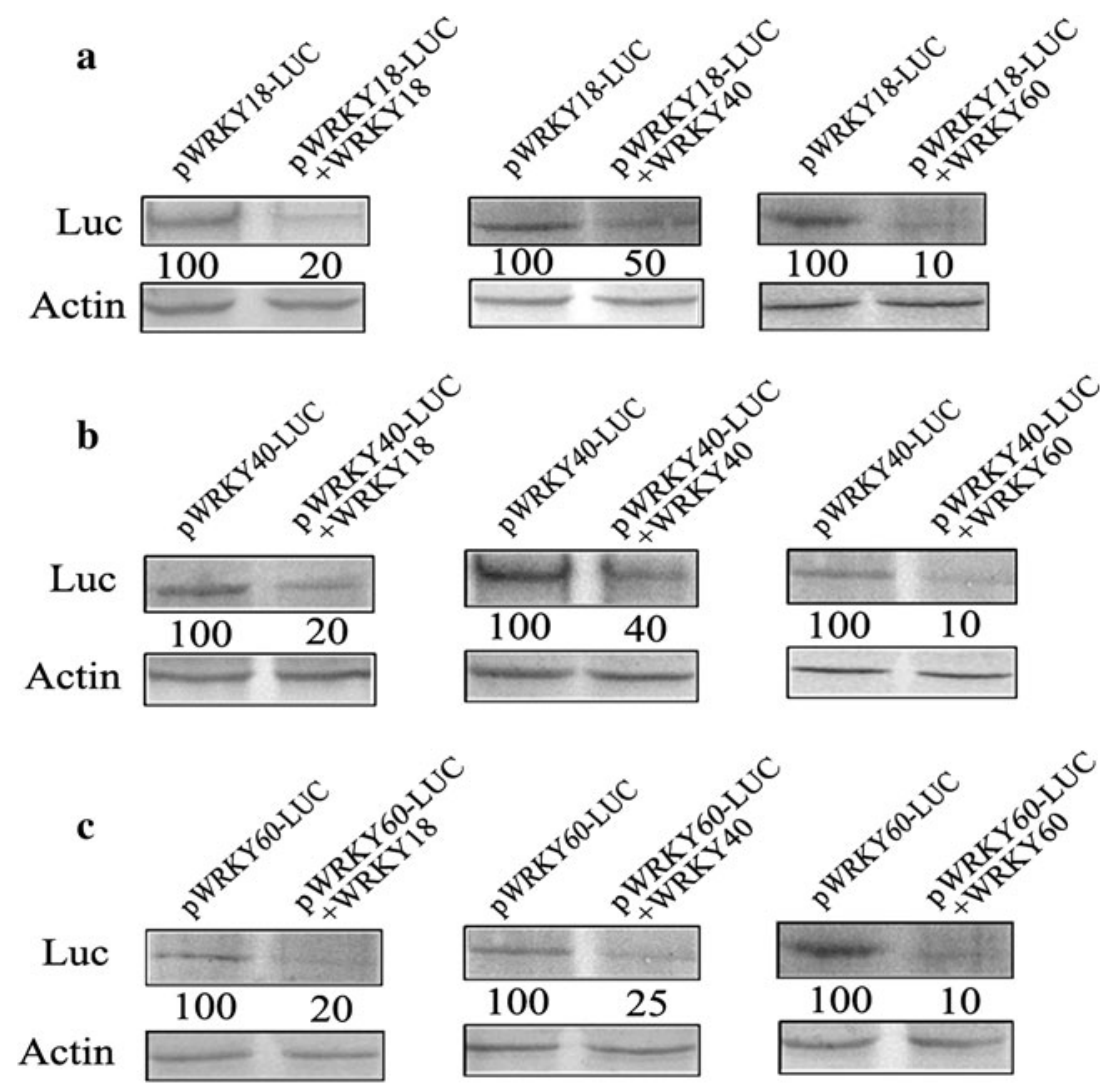

germination growth in response to $\mathrm{ABA}$ as described in the Materials and Methods section. For the mature plants, ABA was applied by spraying plants with ABA solution. We showed that in wild-type plants at the early stage of seedling growth, ABA treatments significantly decreased the expression levels of the three WRKY genes, and that ABA treatments at low concentrations such as 0.5 and $1 \mu \mathrm{M}$ induced significant repressive effects, and such repressive effects appeared to be dependent on ABA dose (Fig. 6a). It is also noteworthy that ABA repressed the WRKY4O gene more strongly than the WRKY18 and WRKY60 genes (Fig. 6a). In the mature wild-type Col plants, ABA treatments repressed all three $W R K Y$ genes in which expression of WRKY18 was first repressed and was partly recovered later, showing a different time course compared with the WRKY40 and WRKY60 genes (Fig. 6b). The level of WRKY40 was lower than that of WRKY18 or WRKY60 at $8 \mathrm{~h}$ after ABA application, which is similar to the data for the early-stage seedlings (Fig. 6a, b). In the mature wrky mutants, we observed the same response patterns to $\mathrm{ABA}$ treatments as seen in wild-type plants, except that in wrky 18 and wrky 40 mutants, the repressive effects of ABA on the WRKY60 gene were attenuated, which was more significant in the wrky 18 wrky40 double mutant (Fig. 6b). These data suggest that the response of the WRKY60 gene to ABA partly requires both WRKY18 and WRKY40, but the ABA response of the WRKY18 or the WRKY4O gene does not require WRKY60.

It should be noted that Chen and others (2010) observed that $\mathrm{ABA}$ treatment at $5 \mu \mathrm{M}$ by spraying 3 week-old mature plants promoted expression of the three WRKY genes. However, we never observed any significant effects of such low-concentration ABA on expression of the WRKY genes for 3 week-old mature plants under our conditions (data not shown), though a significant repressive effect of $\mathrm{ABA}$ at low concentrations $(\leq 10 \mu \mathrm{M})$ was observed for young seedlings at the early developmental stage, as described above.

\section{ABA Hypersensitive Phenotypes of Different wrky Mutants: New Observations}

Given that Chen and others (2010) observed ABA-insensitive phenotypes of wrkyl 18 and wrky60 mutants, which is different than our observations (Shang and others 2010), it is necessary to use multiple testing systems to assess whether WRKY18 and WRKY60 play negative or positive roles in ABA signaling. In our previous report, we assayed the ABA sensitivity of the different wrky mutants in early seedling growth using a technique by which the germinating seeds were transferred $48 \mathrm{~h}$ after stratification from ABA-free medium to the medium containing low levels of 

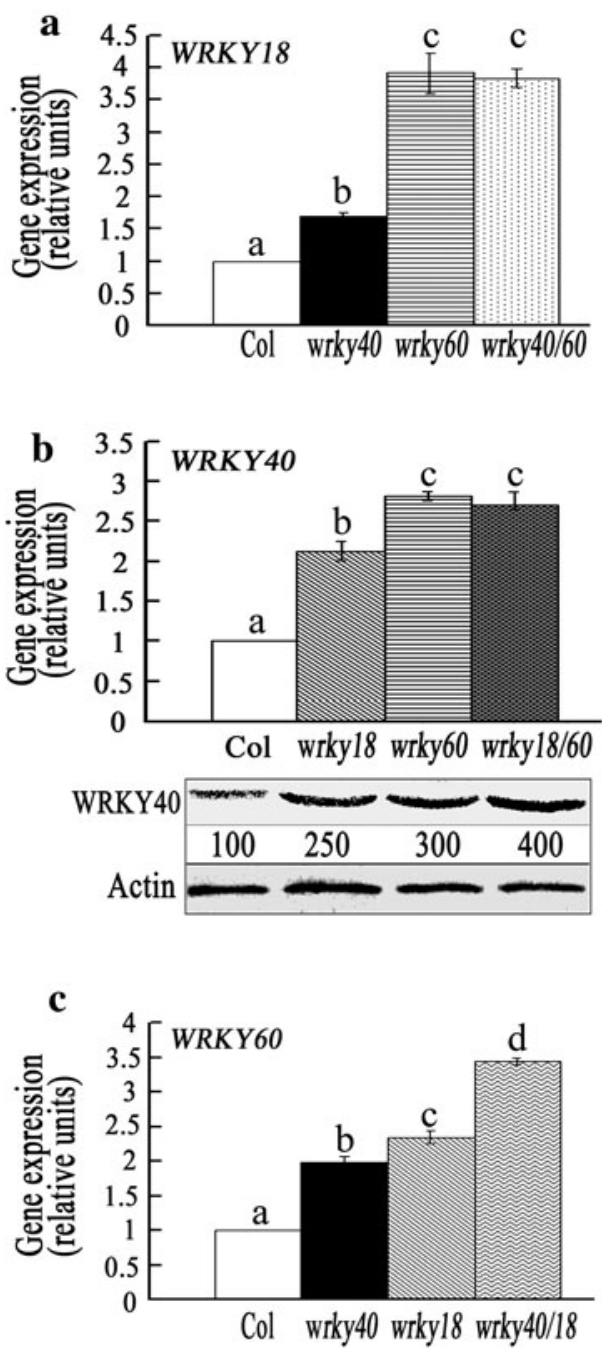

Fig. 5 Expression of WRKY18, WRKY40, and WRKY60 increases in the wrky mutants. Left panels in $\mathbf{a}, \mathbf{b}$, and $\mathbf{c}$ show real-time PCR data (also immunoblotting data for WRKY40) obtained from 3-week-old plants, and right panels show pictures of GUS staining of 3-day-old plants (left) and 3-week-old leaves (right). a Expression of WRKY18 is enhanced in wrky40, wrky60 single mutants, and the wrky40 wrky60 double mutants (left panel), and the activity of the WRKY18 promoter, estimated by the transgenic GUS level, increases in the wrky 18 , wrky 40 , and wrky60 single mutants. Each value is the mean $\pm \mathrm{SE}$ of five independent biological determinations, and different letters indicate significant differences at $P<0.05$ (Duncan's multiple-range test). b Expression of WRKY40, tested by both realtime PCR (top columns) and immunoblotting (bottom, with actin as a

ABA ( $\leq 2 \mu \mathrm{M}$ in general; Shang and others 2010). We first verified our previous findings using the same testing system. We observed that the wrky18, wrky40, and wrky60 single mutants, the wrky 18 wrky 40 double mutant, and the wrky18 wrky40 wrky60 triple mutant showed ABAhypersensitive phenotypes in ABA-induced post-germination growth arrest (Fig. 7a, b). Among these mutants, the wrky 40 single mutant, the wrky 18 wrky 40 double mutant, and the wrkyl8 wrky40 wrky60 triple mutant showed
pWRKY18-GUS

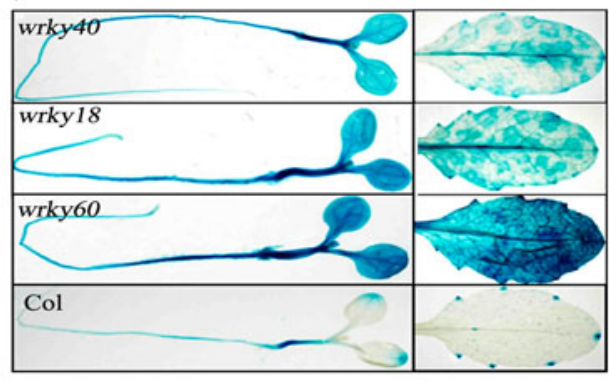

pWRKY40-GUS

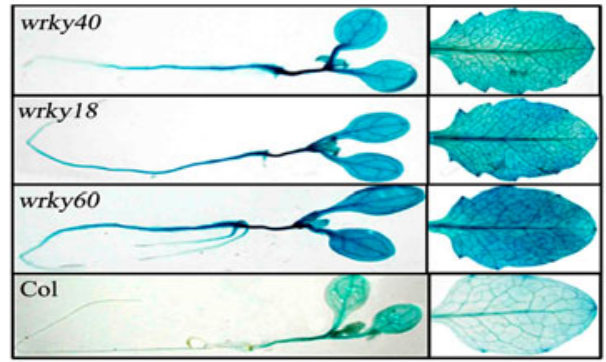

\section{pWRKY60-GUS}

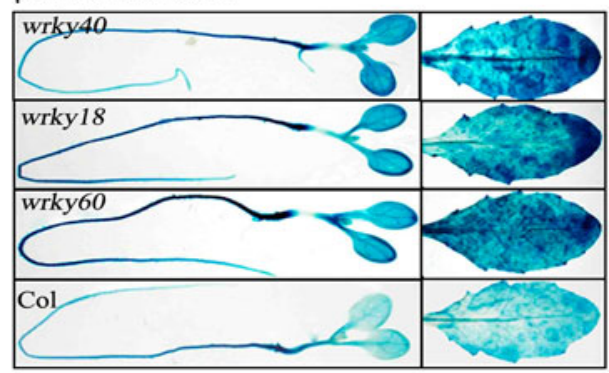

loading control) assays, is enhanced in the wrky18, wrky60 single mutants and the wrky18 wrky60 double mutants (left panel), and the activity of the WRKY40 promoter, estimated by the transgenic GUS level, increases in the wrky18, wrky40, and wrky60 single mutants. WRKY40 protein amounts were evaluated by scanning the protein bands, and relative band intensities, normalized relative to the intensity with the value from the wild-type plant (as $100 \%$ ), are indicated by numbers below the bands. c Expression of WRKY60 is enhanced in the wrky18, wrky40 single mutants and the wrky 18 wrky40 double mutants (left panel), and the activity of the WRKY60 promoter, estimated by the transgenic GUS level, increased in the wrky 18 , wrky 40 , and wrky60 single mutants. Each value presented in the columns is the mean $\pm \mathrm{SE}$ of five independent determinations

stronger and the wrky60 showed weaker ABA-hypersensitive phenotypes (Fig. 7a, b). Additionally, it is noteworthy that the wrky60 mutation attenuated ABA-hypersensitive phenotypes when combined with wrky 18 or wrky 40 (Fig. 7a, b), which is consistent with the previous suggestions that WRKY60 may antagonize WRKY18 and WRKY40 in a complex manner at the transcriptional, translational, and post-translational level (Xu and others 2006; Shang and others 2010). The similar ABA-hypersensitive phenotypes 

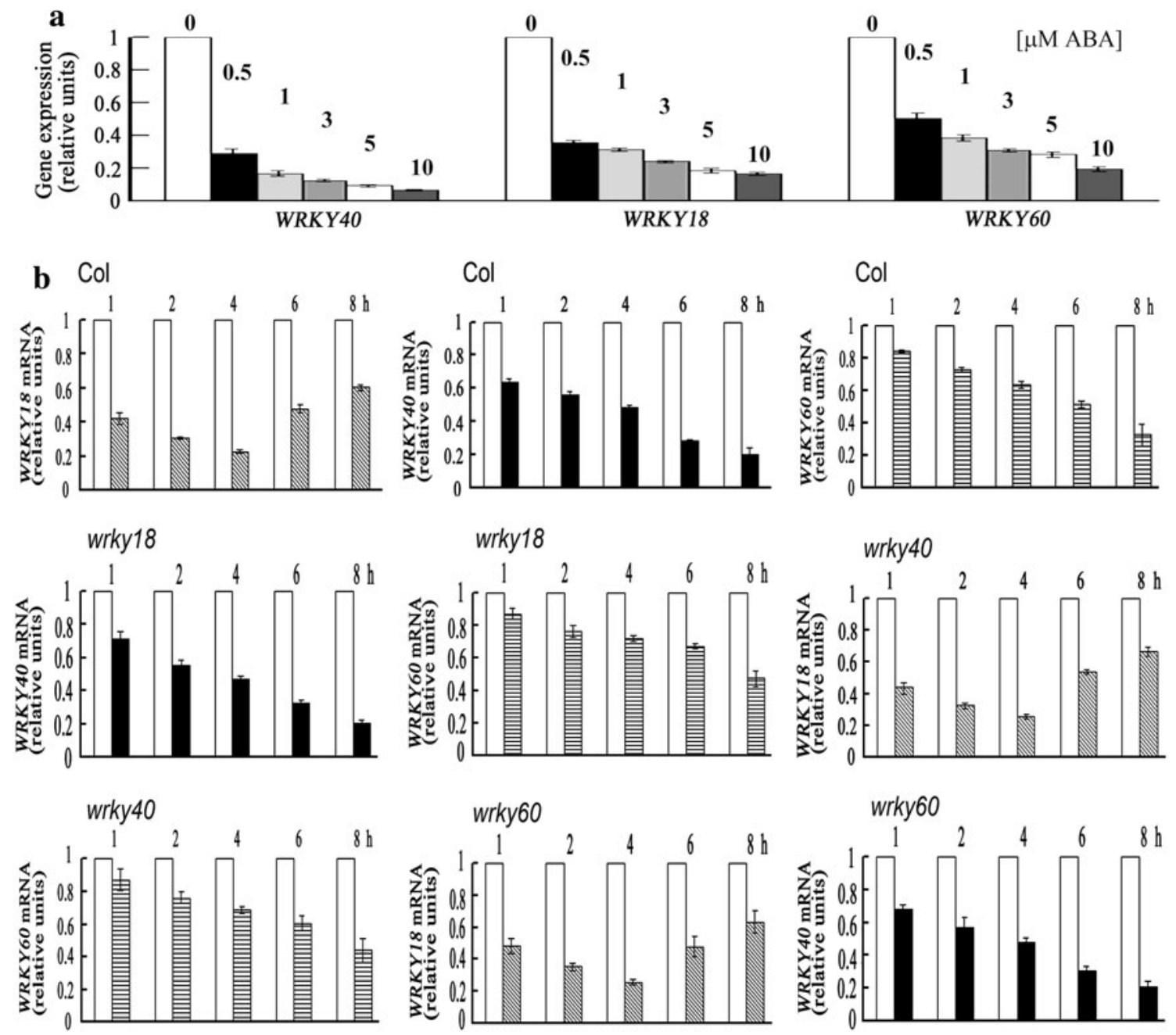

\section{wrky18 wrky40}
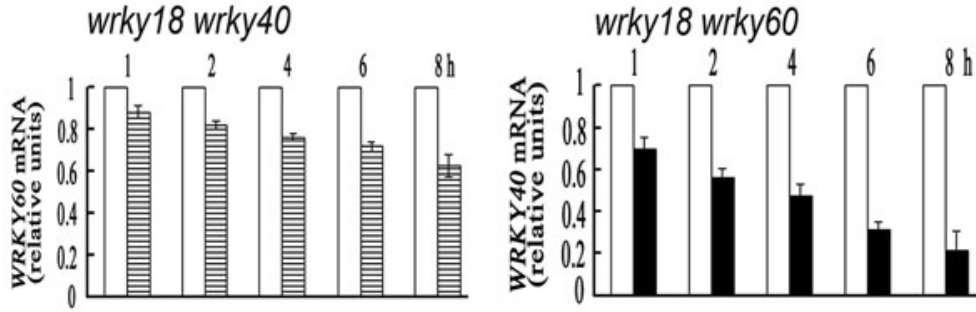

wrky40 wrky60

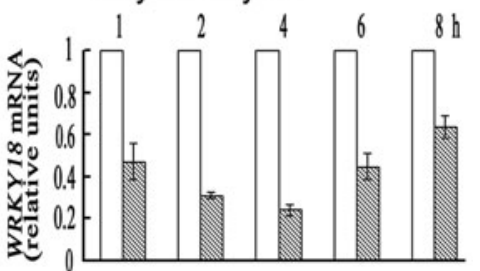

Fig. $6 \mathrm{ABA}$ treatments induce repression of the three $W R K Y$ genes in wild-type plants and wrky mutants. a Exogenous ABA inhibits expression of WRKY18, WRKY40, and WRKY60 genes in wild-type plants. The germinating seeds were transferred $48 \mathrm{~h}$ after stratification into ABA-containing medium (ABA concentrations are indicated above the columns), and the seedlings were sampled for real-time PCR analysis 14 days after transfer. Each value is the mean \pm SE of five independent determinations. b ABA treatments repress the WRKY18, WRKY40, and WRKY60 genes in wild-type plants and wrky mutants. Three-week-old seedlings of the wild-type Col plants and

of these wrky mutants were observed in ABA-induced seed germination inhibition (Fig. 7c). These data are totally consistent with our previous observations (Shang and others 2010). different wrky single (wrky18, wrky40, and wrky60) and double mutants (wrky 18 wrky 40 , wrky 18 wrky60, and wrky40 wrky60) were sprayed with $0 \mu \mathrm{M}$ ABA (white columns) or $100 \mu \mathrm{M}$ ABA (black columns, WRKY40; hatched columns, WRKY18 or WRKY60) and were sampled for real-time PCR analysis $1,2,4,6$, or $8 \mathrm{~h}$ after the ABA treatment. The real-time PCR value obtained from the sample of the ABA-free treatment was taken as 1, and the value from the sample of the ABA treatment was normalized relative to the ABA-free treatment value obtained at the same sampling time. Each value is the mean $\pm \mathrm{SE}$ of five independent determinations

Additionally and importantly, in the present experiment we used two different tests to assay ABA responses of the different wrky mutants in early seedling growth. In one test the seeds were sown directly in ABA-containing medium 

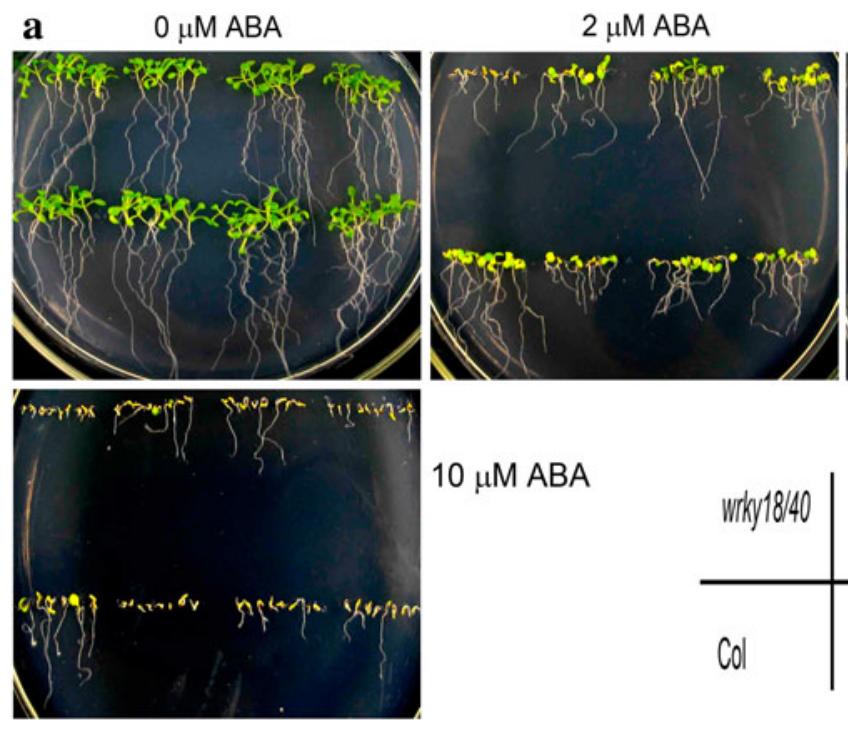

$5 \mu \mathrm{M}$ ABA

$10 \mu \mathrm{M} \mathrm{ABA}$
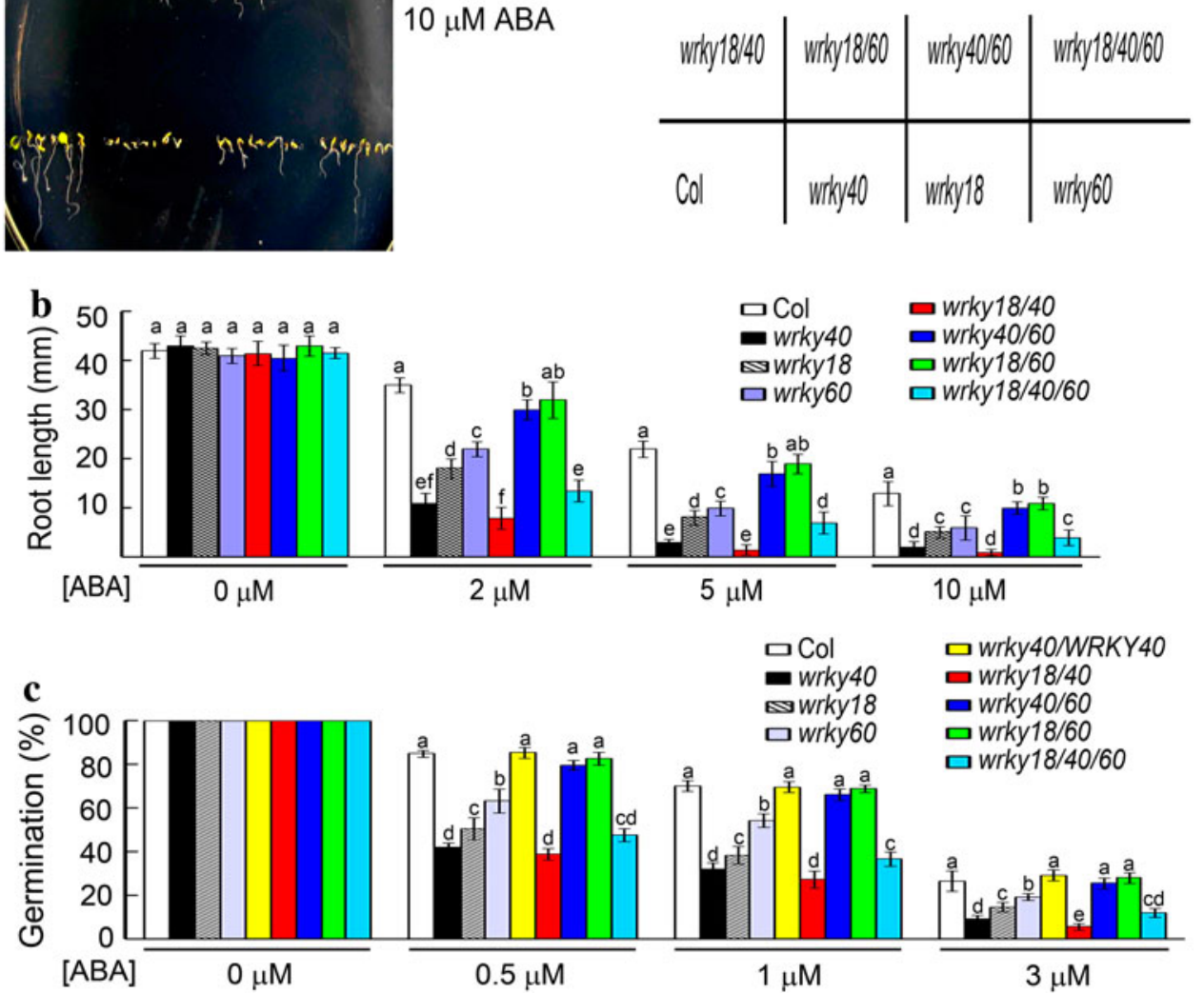

Fig. 7 ABA-hypersensitive phenotypes of different wrky mutants in ABA-induced seed germination inhibition and post-germination growth arrest. a Post-germination growth of wild-type Col plants and the wrky18, wrky40, and wrky60 single mutants, the wrky18 wrky40 (wrky18/40), wrky18 wrky60 (wrky18/60), and wrky40 wrky60 (wrky40/60) double mutants, and the wrky18 wrky40 wrky60 (wrkyl8/ 40/60) triple mutants in the ABA-free medium ( $0 \mu \mathrm{M} \mathrm{ABA})$ and medium containing 2, 5, or $10 \mu \mathrm{M}$ ABA. Post-germination growth was assessed by transferring germinating seeds $48 \mathrm{~h}$ after stratification from ABA-free medium to medium containing ABA. The seedling response to ABA was investigated 12 days after stratification. b The columns show corresponding statistical data of the root length of these mutants described in panel $\mathbf{a}$, in which each value is

and seedling growth was investigated after germination. In the other test the young seedlings were transferred from the ABA-free medium to the medium containing high levels of ABA $(\geq 2 \mu \mathrm{M}) 4$ days (but not $48 \mathrm{~h}$ ) after stratification, which is considered different from the $48 \mathrm{~h}$-seedling transfer system because there exists a short developmental the mean \pm SE of five independent biological determinations and different letters indicate significant differences at $P<0.05$ (Duncan's multiple-range test) when comparing values within the same ABA concentration. $\mathbf{c}$ Seed germination rates of wild-type Col plants and different wrky mutants in the ABA-free medium $(0 \mu \mathrm{M} \mathrm{ABA})$ and medium containing $0.5,1$, or $3 \mu \mathrm{M}$ ABA. Seed germination rate was recorded $48 \mathrm{~h}$ after stratification. The wrky $40 / W R K Y 40$ is a line of the wrky 40 mutant complemented with the WRKY4O cDNA. Each value is the mean $\pm \mathrm{SE}$ of five independent biological determinations and different letters indicate significant differences at $P<0.05$ (Duncan's multiple-range test) when comparing values within the same ABA concentration

window (about $48 \mathrm{~h}$ ) after stratification that is likely associated with early $A B I 5$ expression when the seedlings are more sensitive to ABA (Lopez-Molina and others 2001; $\mathrm{Wu}$ and others 2009; Jiang and $\mathrm{Yu}$ 2009). With these two different systems, we obtained substantially the same results as those obtained previously with the 48 -h-seedling 
transfer system (Figs. 8, 9). This suggests that the WRKYmediated ABA signaling pathway involves more regulators than ABI5. In the testing system in which seeds were sown directly in the ABA-containing medium, we observed that the different wrky mutants, except wrky 18 wrky60 and wrky40 wrky60, showed significant ABA-hypersensitive phenotypes in $\mathrm{ABA}$-induced post-germination growth arrest at low levels of ABA ( 0.4 or $0.6 \mu \mathrm{M}$ ABA, Fig. 8a, b). It is particularly noteworthy that with the 4 day-seedling transfer system, ABA-hypersensitive phenotypes of these wrky mutants in seedling growth were more apparent when ABA concentrations were higher ( $\geq 5 \mu \mathrm{M}$, Fig. 9a, b), especially for the wrky 18 wrky 40 wrky60 triple mutant, which showed ABA hypersensitivity at $10 \mu \mathrm{M}$ ABA (Fig. 9a, b). At $2 \mu \mathrm{M}$ ABA, only the wrky40 mutant showed an ABA-hypersensitive phenotype, whereas other wrky mutants showed a wild-type ABA response (Fig. 9a, b). This is different from the above-mentioned data obtained with the $48 \mathrm{~h}$-seedling transfer system where
ABA-hypersensitive phenotypes of the wrky mutants in seedling growth were significantly induced at lower ABA levels $(\geq 2 \mu \mathrm{M}$, Fig. 7a, b). These observations of ABAhypersensitive phenotypes of the different wrky mutants are inconsistent with those of Chen and others (2010) who showed ABA-insensitive phenotypes of the wrkyl8 and wrky 60 mutants at $2 \mu \mathrm{M} \mathrm{ABA}$ when transferring young seedlings from the ABA-free medium to the medium containing ABA 4 days after stratification. We noted that the wrky 60 and even the wrky 18 mutant showed relatively weaker ABA-hypersensitive phenotypes at $\mathrm{ABA}$ concentrations higher than $2 \mu \mathrm{M}$ (Fig. 9a, b) in the same 4-day-seedling transfer system used by Chen and others (2010), but in no case did these mutants show ABAinsensitive phenotypes (Figs. 7, 8, 9) as described by Chen and others (2010). Consistent with our previous observations (Shang and others 2010), all the data demonstrate that WRKY18, WRKY40, and WRKY60 negatively, not positively, regulate ABA signaling.
Fig. 8 ABA-hypersensitive phenotypes of different wrky mutants in ABA-induced postgermination growth arrest assessed by directly planting seeds in ABA-containing medium. a Post-germination growth of wild-type Col plants and the wrky 18 , wrky 40 , and wrky 60 single mutants, the wrky18 wrky40 (wrky18/40), wrky18 wrky60 (wrky18/60), and wrky 40 wrky60 (wrky40/60) double mutants, and the wrky 18 wrky40 wrky60 (wrky18/40/60) triple mutants in the ABA-free medium $(0 \mu \mathrm{M} \mathrm{ABA})$ and medium containing 0.4 or 0.6 $\mu \mathrm{M}$ ABA. Post-germination growth was investigated 12 days after stratification. b The columns show corresponding statistical data of the root length of these mutants described in panel $\mathbf{a}$, in which each value is the mean $\pm \mathrm{SE}$ of five independent biological determinations and different letters indicate significant differences at $P<0.05$ (Duncan's multiple-range test) when comparing values within the same ABA concentration a

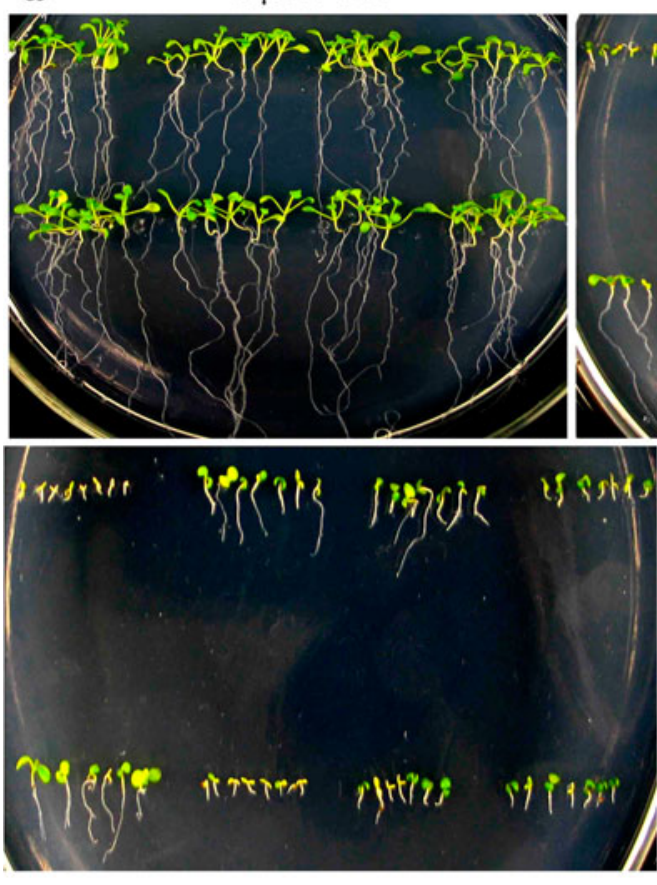

$0 \mu \mathrm{M}$ ABA

$0.4 \mu \mathrm{M}$ ABA

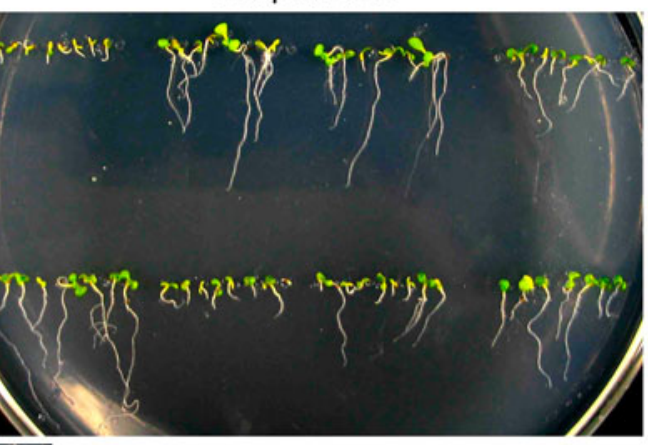

$0.6 \mu \mathrm{M}$ ABA

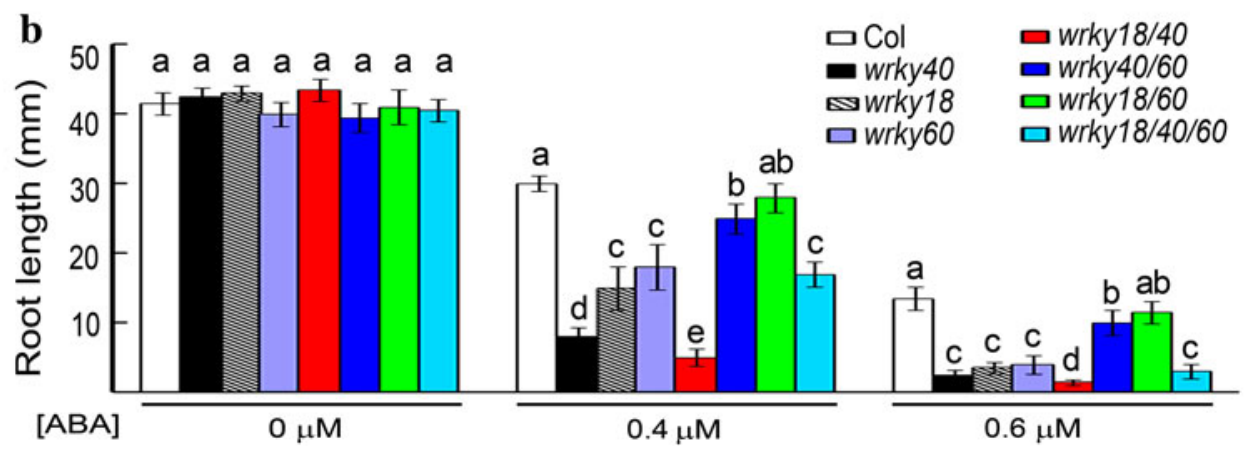


Fig. 9 ABA-hypersensitive phenotypes of different wrky mutants in ABA-induced early seedling growth inhibition assessed by transferring 4-dayold seedlings from ABA-free medium to medium containing ABA. a Seedling growth of the wild-type plants $(\mathrm{Col})$ and different wrky mutants was recorded in the $\mathrm{ABA}$-free medium $(0 \mu \mathrm{M}$ ABA, left in top panels) and medium containing ABA $(2 \mu \mathrm{M}$, right in top panels; $5 \mu \mathrm{M}$, left in bottom panels; 10 $\mu \mathrm{M}$, right in bottom panels) 2 weeks after stratification. b The columns show corresponding statistical data of the root length of these mutants described in panel $\mathbf{a}$, in which each value is the mean \pm SE of five independent biological determinations and different letters indicate significant differences at $P<0.05$

(Duncan's multiple-range test) when comparing values within the same $\mathrm{ABA}$ concentration
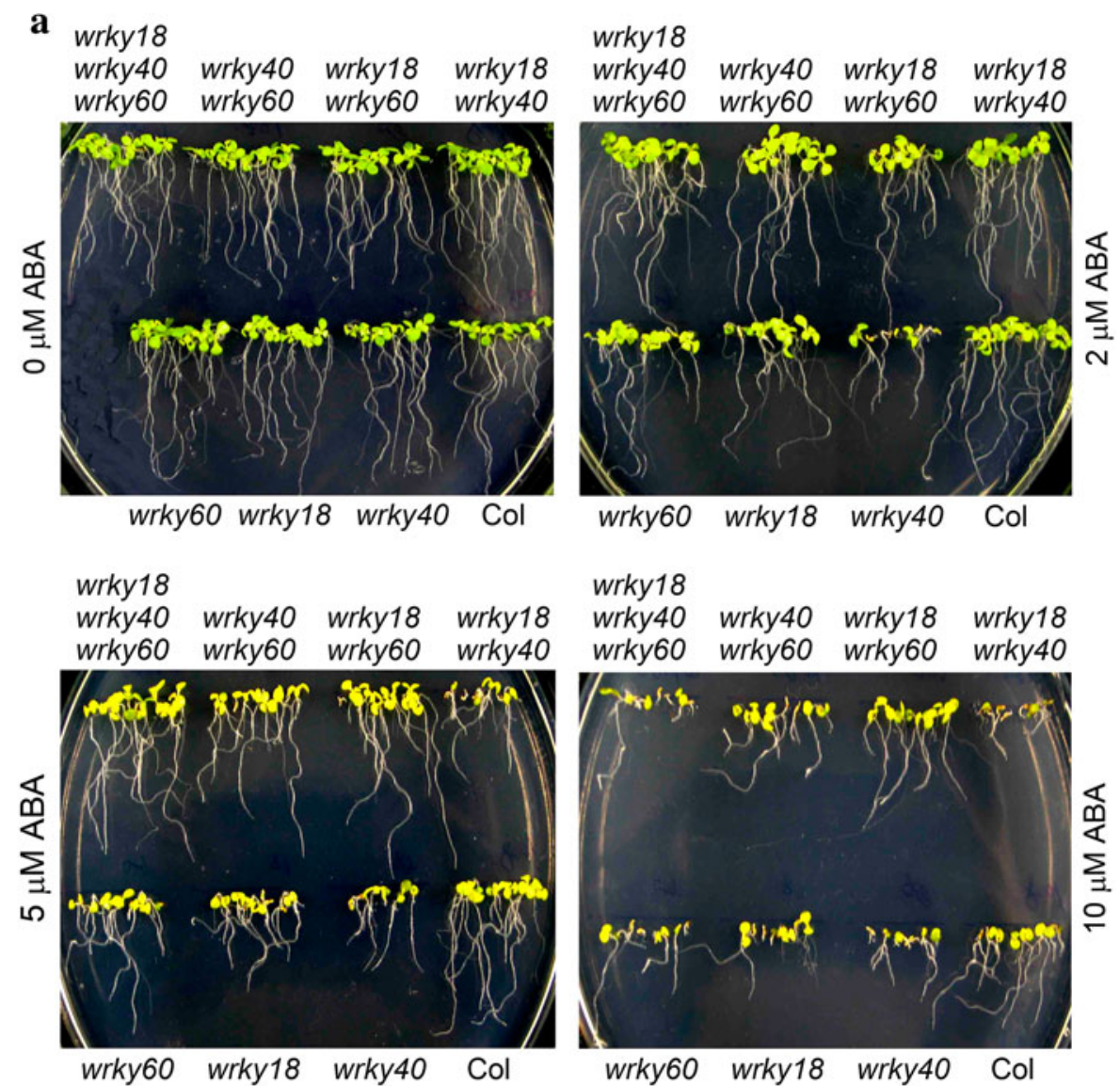

wrky18

wrky40 wrky40 wrky18 wrky18 wrky60 wrky60 wrky60 wrky40
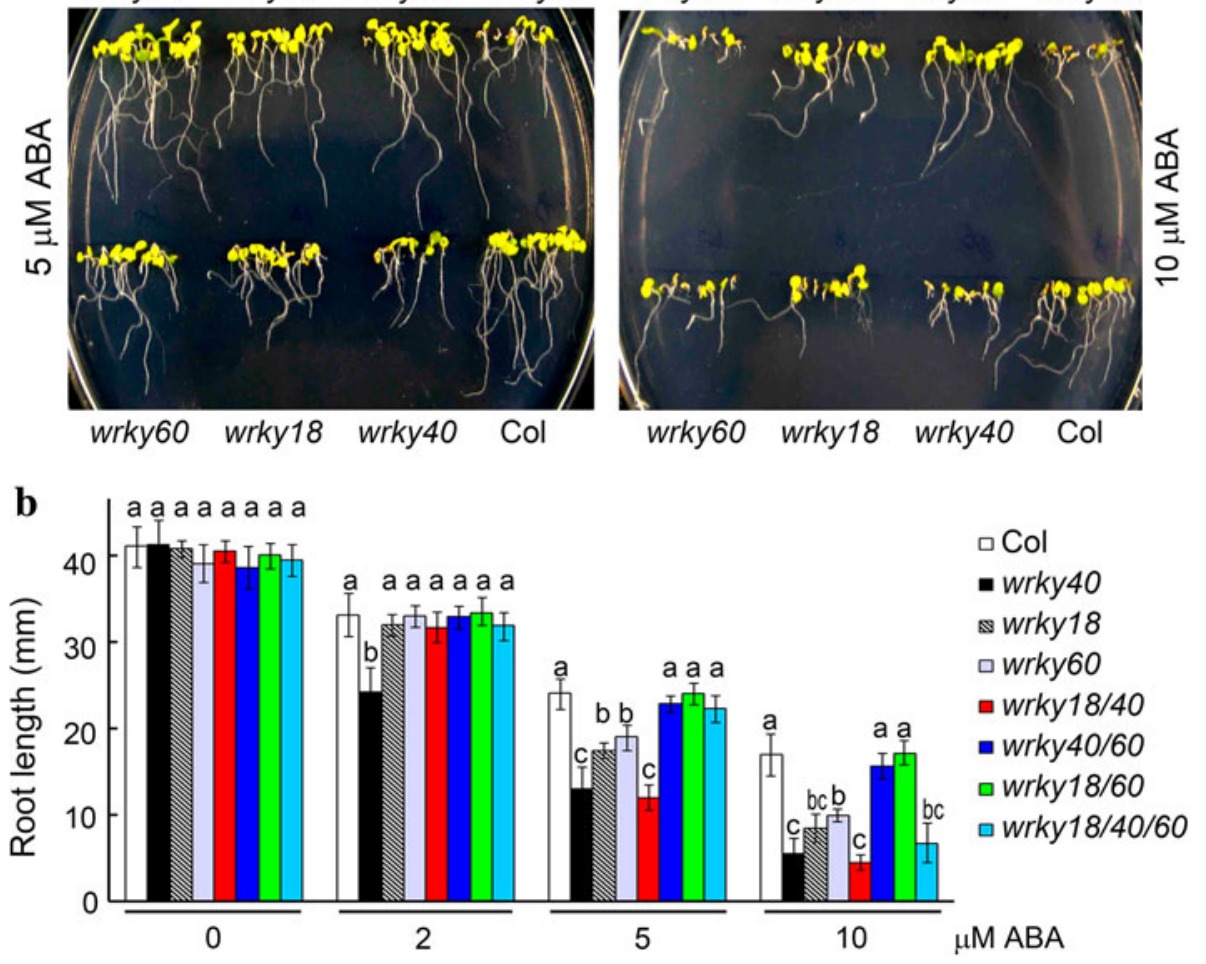

\section{Discussion}

Auto- and Cross-repression of WRKY18, WRKY40, and WRKY60 Suggests a Sophisticated Mechanism to Maintain Homeostasis of the WRKY Transcription Repressors in ABA Signaling

Although it has been known that WRKY18, WRKY40, and WRKY60 are involved in ABA signaling (Chen and others 2010; Shang and others 2010), it remains unclear how the three homologous WRKY transcription factors cooperate to regulate $\mathrm{ABA}$ signaling. In the present study, we provide evidence that the three WRKY proteins target all three $W R K Y$ genes and repress their expression. We first showed that the WRKY18, WRKY40, and WRKY60 proteins directly bind the $\mathrm{W}$-box regions in various domains of the promoters of all three WRKY genes (Fig. 10a), which was evidenced by chromatin immunoprecipitation assays for the WRKY40 protein and by gel shift assays for all three WRKY proteins (Figs. 1, 2). Furthermore, we showed that the three WRKY proteins inhibit expression of all three $W R K Y$ genes, which was evidenced in both an in vivo assay of coexpression of the WRKY proteins with the three WRKY promoters (Figs. 3, 4) and expression analysis of the three $W R K Y$ genes in various wrky mutants (Fig. 5).

As previously reported (Shang and others 2010) and supported in the present study by several lines of new evidence (Figs. 7, 8, 9), the three WRKY transcription 


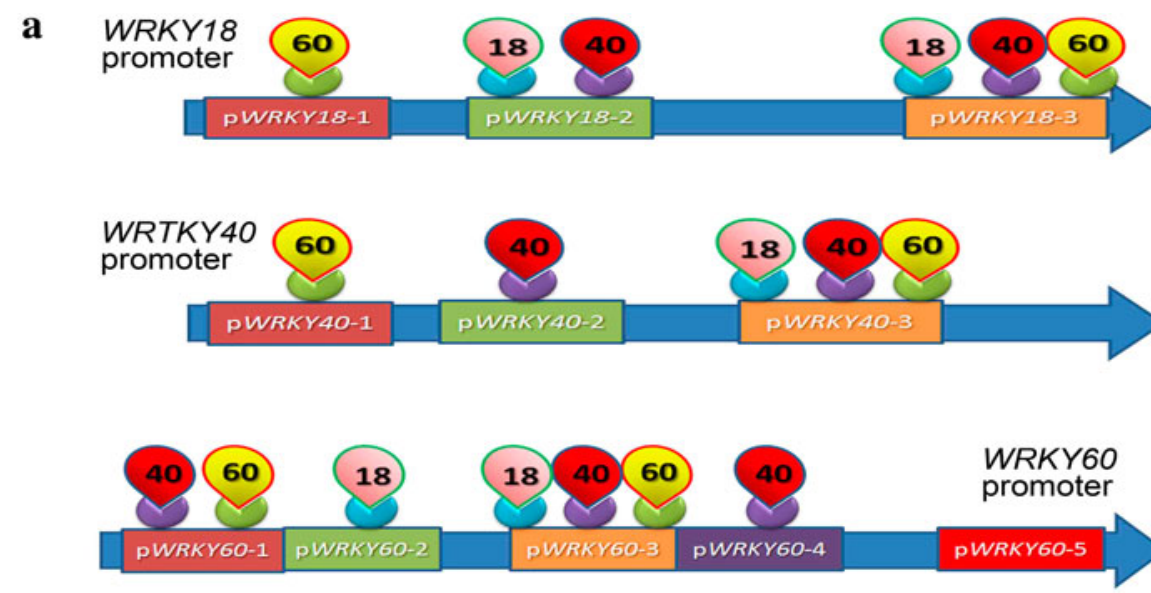

b

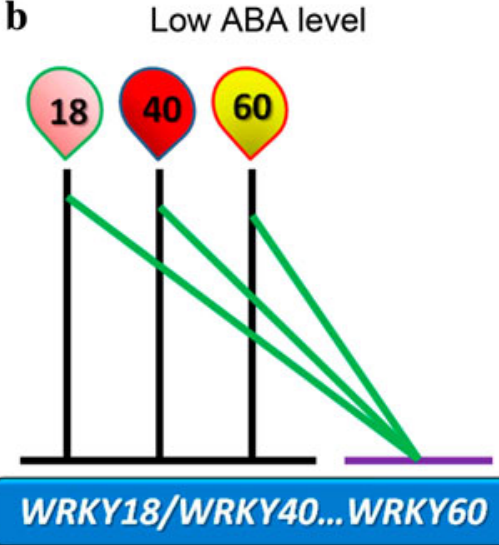

Fig. 10 Proposed working model of auto- and cross-repression of three WRKY genes. a Different binding domains of the three WRKY transcription factors WRKY18 (indicated by 18), WRKY40 (indicated by 40), and WRKY60 (indicated by 60) in the WRKY18 (top), WRKY4O (middle), and WRKY60 (bottom) promoters. See Fig. 1a for description of different domains in the promoters. b Working model of auto- and cross-repression of three WRKY genes. Left panel: Under the conditions of low ABA levels, each of the three WRKY transcription factors represses its own encoding gene and two other

factors function negatively in ABA signaling as transcription repressors. Such transcription repressors inhibit the expression of a set of ABA-responsive genes (Shang and others 2010), which is necessary for plants to avoid growth arrest induced by expression of these ABA-responsive genes and thus keep up their vigorous development under environmental conditions favorable to growth. However, homeostasis of such transcription repressors in plant cells may be of particular importance to balance the repressive effects. The auto- and cross-repression of the three WRKY transcription repressors likely provide a module to construct a sophisticated mechanism to maintain this homeostasis and to balance growth promotion and arrest of growth.

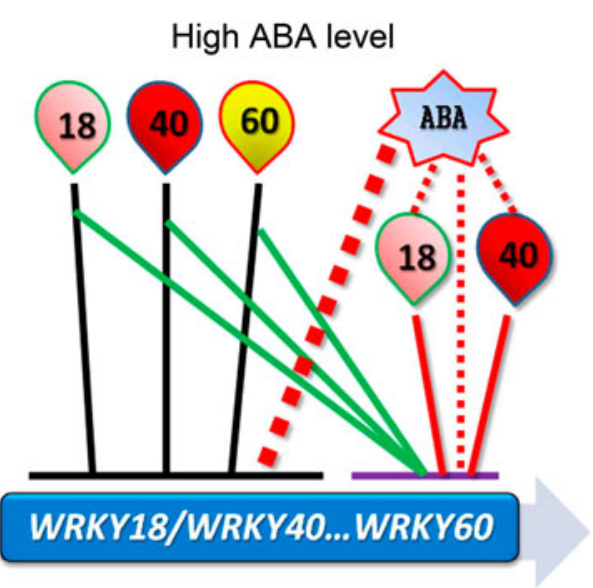

homologous WRKY genes. Right panel: While each of the three WRKYs functions to inhibit expression of three WRKY genes, a high level of ABA participates in repressing WRKY18 and WRKY4O genes by a yet unknown mechanism independent of the three WRKYs, and inhibits WRKY60 expression through a WRKY18/40-dependent and a WRKY18/40-independent pathway. Bars indicate repression. Line denotes direct effect and dotted line indirect effect. See the text for detailed explanation

We previously observed that $\mathrm{ABA}$ treatment inhibits expression of the WRKY4O gene (Shang and others 2010). In the present experiments, we confirmed this observation and furthermore showed that all three WRKY genes were repressed by exogenous $\mathrm{ABA}$ in both early and mature stages of plant growth (Fig. 6). Interestingly, we found that in different wrky mutants, ABA treatment repressed all three $W R K Y$ genes with the same $\mathrm{ABA}$ response patterns as in wild-type plants, indicating that ABA induces inhibition of the WRKY genes through a mechanism at least partly independent of the WRKY proteins. However, the response of the WRKY60 gene to ABA was shown to partly require WRKY18 and WRKY40 (Fig. 6), suggesting that a 
complex mechanism was involved in the response of the $W R K Y$ genes to ABA.

These findings allow us to propose a working model of auto- and cross-repression of the three WRKY genes in ABA signaling (Fig. 10b). Under the environmental conditions favorable to growth with low ABA levels, each of the three WRKY transcription factors represses its own encoding gene and two other homologous WRKY genes. This repressive effect may balance some unknown transcription activation processes and results in homeostasis of the WRKY proteins. In stressful conditions unfavorable to plant growth, high levels of ABA participate in repressing WRKY18 and WRKY4O genes by a yet unknown mechanism independent of the three WRKY proteins, and inhibit WRKY60 expression through both a WRKY18/40-dependent and a WRKY18/40-independent pathway. The ABAinduced repression of the WRKY genes works together with the auto- and cross-repression mechanisms of the WRKY genes and thus strongly antagonizes the transcription activation process. This double-repressive effect creates a new homeostasis of lower levels of the WRKY proteins to relieve ABA-responsive genes of inhibition and to induce physiological responses.

WRKY18, WRKY40, and WRKY60 Are Negative, not Positive, ABA Signaling Regulators

In contrast with the findings of Chen and others (2010) who considered WRKY18 and WRKY60 positive regulators of ABA signaling, we provide new evidence that all three WRKY transcription factors negatively, not positively, regulate ABA signaling. In this signaling event, WRKY40 likely plays a more important role than WRKY18 and WRKY60, and WRKY60 appears to antagonize WRKY18 and WRKY40 (Figs. 7, 8, 9). These findings are consistent with our previous observations (Shang and others 2010).

We observed that the ABA-hypersensitive phenotype of the wrky 40 mutant is stronger, but that of the wrky18 and wrky60 mutants, though stable and clear, is relatively weaker (Figs. 7, 8, 9). Such a weak phenotype may be affected more easily by the environmental conditions of plant growth, which affect seed maturation and seed quality and thus cause different responses to ABA during early developmental stages. Additionally, postharvest storage conditions of seeds, including storage time before use and environment during storage, may also significantly affect seed response to ABA. This may partly explain the observation by Chen and others (2010) of ABAhypersensitive phenotypes in the wrky 40 mutant but no such observable phenotype in the wrky18, wrky60 single mutants and the relative double mutants. The molecular mechanism by which WRKY40 plays a central role, but WRKY18 and WRKY60 function as weak partners, remains unknown. However, it has been known that the WRKY transcription factor family consists of numerous members, in which several members may be positive and some others may be negative ABA signaling regulators. These positive and negative regulators may antagonize each other or function redundantly. For instance, two other members of the WRKY family, WRKY2 and WRKY63, have been identified as additional transcription repressors negatively involved in ABA signaling (Jiang and Yu 2009; Ren and others 2010). The possible antagonistic effects among WRKY members and their functional redundancy may be responsible for the observed weak ABA-hypersensitive phenotypes of the wrky18 and wrky60 mutants. The different environmental conditions during seed maturation and storage may have an impact on the weak phenotypes of ABA sensitivity of the wrky 18 and wrky 60 mutants, thus possibly resulting in the discrepancies. In the present experiments, however, we verified our previous observations using different testing systems to avoid possible errors at three different growth stages with different concentrations of ABA: with low levels of ABA ( 0.4 and $0.6 \mu \mathrm{M})$ since stratification (before germination, Fig. 8) and with high levels of $\mathrm{ABA}(2,5$, and $10 \mu \mathrm{M})$ since the germination stage ( $48 \mathrm{~h}$ after stratification, Fig. 7 ) or the young seedling stage (4 days after stratification, Fig. 9). All our observations allow us to conclude that WRKY18, WRKY40, and WRKY60 negatively, not positively, regulate ABA signaling. We are working to identify more members of the Arabidopsis WRKY family as ABA signaling regulators to assess whether the WRKY transcription factors play both negative and positive roles in ABA signaling, which will help us to understand the complicated ABA signaling pathways.

Acknowledgments This research was supported by the National Key Basic Research Program of China (2012CB114300-002), the National Natural Science Foundation of China (grant Nos. 90817104 and 31170268), and the Foundation for the Author of National Excellent Doctoral Dissertation of China (Grant No. 201065).

Open Access This article is distributed under the terms of the Creative Commons Attribution License which permits any use, distribution, and reproduction in any medium, provided the original author(s) and the source are credited.

\section{References}

Adie BAT, Perez-Perez J, Perez-Perez MM, Godoy M, SanchezSerrano JJ, Schmelz EA, Solanoa R (2007) ABA is an essential signal for plant resistance to pathogens affecting JA biosynthesis and the activation of defenses in Arabidopsis. Plant Cell 19:1665-1681

Chen H, Lai ZB, Shi JW, Xiao Y, Chen ZX, Xu XP (2010) Roles of Arabidopsis WRKY18, WRKY40 and WRKY60 transcription factors in plant responses to abscisic acid and abiotic stress. BMC Plant Biol 10:281 
Chen L, Song Y, Li S, Zhang L, Zou C, Yu D (2012) The role of WRKY transcription factors in plant abiotic stresses. Biochim Biophys Acta 1819:120-128

Ciolkowski I, Wanke D, Birkenbihl RP, Somssich I (2008) Studies on DNA-binding selectivity of WRKY transcription factors lend structural clues into WRKY-domain function. Plant Mol Biol 68:81-92

Cutler SR, Rodriguez PL, Finkelstein RR, Abrams SR (2010) Abscisic acid: emergence of a core signaling network. Annu Rev Plant Biol 61:651-679

Eulgem T, Somssich IE (2007) Networks of WRKY transcription factors in defense signaling. Curr Opin Plant Biol 10:366-371

Eulgem T, Rushton PJ, Robatzek S, Somssich IE (2000) The WRKY superfamily of plant transcription factors. Trends Plant Sci 5:199-206

Finkelstein RR, Rock C (2002) Abscisic acid biosynthesis and signaling. In: Somerville CR, Meyerowitz EM (eds), The Arabidopsis Book. Rockville, MD: American Society of Plant Biologists, doi/10.1199/tab.0058. Available at http://www. aspb.org/publications/arabidopsis/

Jefferson RA, Kavanagh TA, Bevan MW (1987) GUS fusions: $\beta$ glucuronidase as a sensitive and versatile gene fusion marker in higher plants. EMBO J 20:3901-3907

Jiang W, Yu D (2009) Arabidopsis WRKY2 transcription factor mediates seed germination and post-germination arrest of development by abscisic acid. BMC Plant Biol 9:96-109

Jones JDG, Dang JL (2006) The plant immune system. Nature 444:323-329

Lopez-Molina L, Mongrand S, Chua NH (2001) A post germination developmental arrest checkpoint is mediated by abscisic acid and requires the ABI5 transcription factor in Arabidopsis. Proc Natl Acad Sci USA 98:4782-4787

Mukhopadhyay A, Deplancke B, Walhout AJM, Tissenbaum HA (2008) Chromatin immunoprecipitation (ChIP) coupled to detection by quantitative real-time PCR to study transcription factor binding to DNA in Caenorhabditis elegans. Nat Protoc 3:698-709

Pandey SP, Somssich IE (2009) The role of WRKY transcription factors in plant immunity. Plant Physiol 150:1648-1655

Ren XZ, Chen ZZ, Liu Y, Zhang HR, Zhang M, Liu Q, Hong XH, Zhu JK, Gong ZZ (2010) ABO3, a WRKY transcription factor, mediates plant responses to abscisic acid and drought tolerance in Arabidopsis. Plant J 63:417-429

Rushton PJ, Torres JT, Parniske M, Wernert P, Hahlbrock K, Somssich IE (1996) Interaction of elicitor-induced DNA binding proteins with elicitor response elements in the promoters of parsley $P R 1$ genes. EMBO J 15:5690-5700

Rushton PJ, Somssich IE, Ringler P, Shen QJ (2010) WRKY transcription factors. Trends Plant Sci 15:247-258

Rushton DL, Tripathi P, Rabara RC, Lin J, Ringler P, Boken AK, Langum TJ, Smidt L, Boomsma DD, Emme NJ, Chen X, Finer JJ, Shen QJ, Rushton PJ (2012) WRKY transcription factors: key components in abscisic acid signaling. Plant Biotech J 10:2-11

Saleh A, Alvarez-Venegas R, Avramova Z (2008) An efficient chromatin immunoprecipitation (ChIP) protocol for studying histone modifications in Arabidopsis plants. Nat Protoc 3:10181025

Shang Y, Yan L, Liu ZQ, Cao Z, Mei C, Xin Q, Wu FQ, Wang XF, Du SY, Jiang T, Zhang XF, Zhao R, Sun HL, Liu R, Yu YT, Zhang DP (2010) The Mg-chelatase H subunit of Arabidopsis antagonizes a group of transcription repressors to relieve ABAresponsive genes of inhibition. Plant Cell 22:1909-1935

Shen YY, Wang XF, Wu FQ, Du SY, Cao Z, Shang Y, Wang XL, Peng CC, Yu XC, Zhu SY, Fan RC, Xu YH, Zhang DP (2006) The Mg-chelatase $\mathrm{H}$ subunit is an abscisic acid receptor. Nature 443:823-826

Ulker B, Somssich IE (2004) WRKY transcription factors: from DNA binding towards biological function. Curr Opin Plant Biol 7:491-498

Wu FQ, Xin Q, Cao Z, Liu ZQ, Du SY, Mei C, Zhao CX, Wang XF, Shang Y, Jiang T, Zhang XF, Yan L, Zhao R, Cui ZN, Liu R, Sun HL, Yang XL, Su Z, Zhang DP (2009) The Mg-chelatase H subunit binds abscisic acid and functions in abscisic acid signaling: new evidence in Arabidopsis. Plant Physiol 150:19401954

Xu X, Chen C, Fan B, Chen Z (2006) Physical and functional interactions between pathogen-induced Arabidopsis WRKY18, WRKY40, and WRKY60 transcription factors. Plant Cell 18: $1310-1326$ 